\title{
Beurling Zeta Functions, Generalised Primes, and Fractal Membranes
}

\author{
Titus W. Hilberdink and Michel L. Lapidus ${ }^{1}$
}

\begin{abstract}
We study generalised prime systems $\mathcal{P}\left(1<p_{1} \leq p_{2} \leq \cdots\right.$, with $p_{j} \in \mathbb{R}$ tending to infinity) and the associated Beurling zeta function $\zeta_{\mathcal{P}}(s)=\prod_{j=1}^{\infty}\left(1-p_{j}^{-s}\right)^{-1}$. Under appropriate assumptions, we establish various analytic properties of $\zeta_{\mathcal{P}}(s)$, including its analytic continuation and we characterise the existence of a suitable generalised functional equation. In particular, we examine the relationship between a counterpart of the Prime Number Theorem (with error term) and the properties of the analytic continuation of $\zeta_{\mathcal{P}}(s)$. Further we study 'well-behaved' g-prime systems, namely, systems for which both the prime and integer counting function are asymptotically well-behaved. Finally, we show that there exists a natural correspondence between generalised prime systems and suitable orders on $\mathbb{N}^{2}$.

Some of the above results may be relevant to the second author's theory of 'fractal membranes', whose spectral partition functions are precisely given by Beurling zeta functions.
\end{abstract}

\section{$\S 1$. Introduction}

\subsection{Generalised primes and Beurling zeta functions}

A generalised prime system $\mathcal{P}$ is a sequence of positive reals $p_{1}, p_{2}, p_{3}, \ldots$ satisfying

$$
1<p_{1} \leq p_{2} \leq \cdots \leq p_{n} \leq \cdots
$$

and for which $p_{n} \rightarrow \infty$ as $n \rightarrow \infty$. From these can be formed the system $\mathcal{N}$ of generalised integers or Beurling integers; that is, the numbers of the form

$$
p_{1}^{a_{1}} p_{2}^{a_{2}} \ldots p_{k}^{a_{k}}
$$

where $k \in \mathbb{N}$ and $a_{1}, \ldots, a_{k} \in \mathbb{N}_{0} \cdot{ }^{2}$ For simplicity, we shall often just refer to g-primes and g-integers. This system generalises the notion of prime numbers and the natural numbers obtained from them. Such systems were first introduced by Beurling [4] and have been studied by numerous authors since then (see, in particular, the papers by Bateman and Diamond [2, Diamond [8], 9], 10], 11], Hall [15], 16], Malliavin [35, Nyman [36] and Lagarias [22]).

\footnotetext{
${ }^{1}$ The work of M. L. Lapidus was partially supported by the U. S. National Science Foundation under grant DMS-0070497.

${ }^{2}$ Here and henceforth, $\mathbb{N}=\{1,2,3, \ldots\}$ and $\mathbb{N}_{0}=\mathbb{N} \cup\{0\}$.

2000 Mathematics Subject Classification. Primary: 11M41, 11N80. Secondary: 11M06, 11N05, 11S45.

Key words and phrases. Beurling (or generalised) primes and zeta functions, Euler product, analytic continuation, functional equation, Prime Number Theorem (with error term), partial orders on prime powers.
} 
Define the counting functions $\pi_{\mathcal{P}}(x)$ and $N_{\mathcal{P}}(x)$ by

$$
\begin{aligned}
& \pi_{\mathcal{P}}(x)=\sum_{p \leq x, p \in \mathcal{P}} 1, \\
& N_{\mathcal{P}}(x)=\sum_{n \leq x, n \in \mathcal{N}} 1 .
\end{aligned}
$$

Here, as elsewhere in the paper, we write $\sum_{p \in \mathcal{P}}$ to mean a sum over all the g-primes, counting multiplicities. Similarly for $\sum_{n \in \mathcal{N}}$. Much of the research on this subject has been about connecting the asymptotic behaviour of the g-prime counting function (1.1) and of the g-integer counting function (1.2) as $x \rightarrow \infty$. Specifically, given the asymptotic behaviour of $\pi_{\mathcal{P}}(x)$, what can be said about the behaviour of $N_{\mathcal{P}}(x)$, and vice versa.

Many of the known results involve the associated zeta function, often referred to as a Beurling zeta function in the literature, which we define formally by the Euler product

$$
\zeta_{\mathcal{P}}(s)=\prod_{p \in \mathcal{P}} \frac{1}{1-p^{-s}} .
$$

This infinite product may be formally multiplied out to give the Dirichlet series

$$
\zeta_{\mathcal{P}}(s)=\sum_{n \in \mathcal{N}} \frac{1}{n^{s}}
$$

We are generally interested in those systems for which $\pi_{\mathcal{P}}(x)=O\left(x^{A}\right)$ for some $A>0$. For then, the series $\sum_{p} p^{-s}$ converges in a half-plane $\{s \in \mathbb{C}: \Re s>\alpha\}$ for some $\alpha \geq 0$. The number $\alpha$ is the abscissa of convergence of this series and also of the Euler product for $\zeta_{\mathcal{P}}(s)$ in $(1.3)$ and of the Dirichlet series $\left(1.3^{\prime}\right)$. To see this, note that for $s$ real and positive,

$$
\sum_{p \leq x} \frac{1}{p^{s}} \leq \sum_{n \leq x} \frac{1}{n^{s}} \leq \exp \left\{\sum_{p \leq x} \log \frac{1}{1-p^{-s}}\right\} \leq \exp \left\{A \sum_{p \leq x} \frac{1}{p^{s}}\right\}
$$

for some positive constant $A$. Hence if one of the series converges as $x \rightarrow \infty$, then so does the other. We shall further avoid the case $\alpha=0$ for the reason that it is quite unlike the standard primes and would moreover require quite different methods to study. Finally, we can renormalise the case $\alpha \in(0, \infty)$ to $\alpha=1$ by defining a new system $\mathcal{P}^{\prime}=\left\{p^{\alpha}: p \in \mathcal{P}\right\}$.

We shall usually drop the reference to $\mathcal{P}$ and $\mathcal{N}$ if there is no chance of ambiguity. Thus from now on we refer to $\pi(x), N(x), \zeta(s)$ etc. to denote the functions $\pi_{\mathcal{P}}(x), N_{\mathcal{P}}(x), \zeta_{\mathcal{P}}(s)$.

Note that in the case when $\mathcal{P}$ is the set of (rational) primes, and hence $\mathcal{N}$ is the set of natural numbers, then $\zeta(s)$ coincides with the classical Riemann zeta function (see, e.g., [13], 20], 41]); further, $\pi(x)$ (resp. $N(x))$ is just the standard prime (resp. integer) counting function.

Next we briefly discuss the content of the rest of this paper. First we recall some of the known results about generalised primes (or integers) and Beurling zeta functions. We close $\S 1$ by briefly explaining the connections between Beurling zeta functions (or g-prime systems) and 'fractal membranes'. ${ }^{3}$

\footnotetext{
${ }^{3}$ This last subsection can be omitted on a first reading.
} 
In $\S 2$, we examine the relationship between a counterpart of the Prime Number Theorem in this context and the properties of the analytic continuation of $\zeta(s)$; we also consider a related question for the integer counting function. Further, we study 'well-behaved' generalised prime systems, namely, systems for which (roughly speaking), both the prime and integer counting functions are asymptotically well-behaved.

In $\S 3$, we consider the analytic continuation of $\zeta(s)$, and examine when it can be 'completed' to satisfy a suitable generalised functional equation. We do not give a complete answer to the latter difficult question, but indicate several approaches and give a criterion for the existence of such a functional equation.

Finally, in $\S 4$, we show that there is a natural one-to-one correspondence between generalised prime systems and suitable orders on $\mathbb{N}^{2}$.

\subsection{Summary of relevant known results}

In this section we give a brief synopsis of the known results relating the asymptotic behaviours as $x \rightarrow \infty$ of $\pi(x), N(x)$, and of some of the properties of the Beurling zeta function $\zeta(s)$. We start with the connections between $\pi(x)$ and $N(x)$.

The research has concentrated on finding conditions for which results of the form

$$
N(x)=a x+E_{1}(x) \quad \Longleftrightarrow \pi(x)=\operatorname{li}(x)+E_{2}(x)
$$

hold. Here $a$ is a positive constant, $\operatorname{li}(x)$ is the logarithmic integral given by

$$
\operatorname{li}(x)=\lim _{\varepsilon \rightarrow 0^{+}}\left(\int_{0}^{1-\varepsilon}+\int_{1+\varepsilon}^{x}\right) \frac{d t}{\log t},
$$

and $E_{1}(x)$ and $E_{2}(x)$ are error terms of smaller order than $x$ and li $(x)$, respectively. The error terms which have been studied (and seem to occur naturally) are of three types; namely, those of the form

$$
O\left(\frac{x}{(\log x)^{\gamma}}\right), O\left(x e^{-c(\log x)^{\alpha}}\right) \quad \text { and } \quad O\left(x^{\theta}\right)
$$

where $\gamma>1, c>0$ and $\alpha, \theta \in(0,1)$.

- Beurling ([4, 1937) showed that

$$
N(x)=a x+O\left(\frac{x}{(\log x)^{\gamma}}\right) \quad \text { for some } \gamma>3 / 2 \text { implies } \quad \pi(x) \sim \frac{x}{\log x},{ }^{4}
$$

which is an analogue (in this more general context) of the Prime Number Theorem. Furthermore, he showed by example that this is false in general for $\gamma=3 / 2$. Conversely, it follows from Diamond's work ([11], Theorem 2) that

$$
\pi(x)=\frac{x}{\log x}+O\left(\frac{x}{(\log x)^{1+\delta}}\right) \quad \text { for some } \delta>0 \text { implies } \quad N(x) \sim a x .
$$

\footnotetext{
${ }^{4}$ Here and henceforth, all such statements are implicitly assumed to be asymptotic as $x \rightarrow \infty$. Moreover, by $f(x) \sim g(x)$, we mean $f(x) / g(x) \rightarrow 1$ as $x \rightarrow \infty$.
} 
- Nyman ([36, 1949) showed that

$$
N(x)=a x+O\left(\frac{x}{(\log x)^{A}}\right) \quad(\forall A) \quad \Longleftrightarrow \pi(x)=\operatorname{li}(x)+O\left(\frac{x}{(\log x)^{A}}\right)
$$

- Malliavin ([35, 1961) showed that

$$
N(x)=a x+O\left(x e^{-c_{1}(\log x)^{\alpha}}\right)
$$

for some $\alpha \in(0,1)$ and $c_{1}>0$, implies

$$
\pi(x)=\operatorname{li}(x)+O\left(x e^{-c_{2}(\log x)^{\beta}}\right),
$$

for some $c_{2}>0$, where $\beta=\alpha / 10$. Hall ([16], 1971) improved this to $\beta=\alpha / 7.91$. Conversely, Malliavin ([35], 1961) showed that if (1.5) holds for some $\beta \in(0,1)$ and $c_{2}>0$, then (1.4) holds for some $a, c_{1}>0$ and $\alpha=\frac{\beta}{2+\beta}$. Diamond $(9,1970)$ improved this to $\alpha=\frac{\beta}{1+\beta}$, and furthermore, Diamond's result contains $\log x \log \log x$ instead of $\log x$ in the exponent.

- Landau ([23, 1903) proved that

$$
N(x)=a x+O\left(x^{\theta}\right) \quad \text { for some } \theta<1
$$

implies

$$
\pi(x)=\operatorname{li}(x)+O\left(x e^{-c \sqrt{\log x}}\right) \quad \text { for some } c>0 .
$$

Diamond, Montgomery and Vorhauer have recently shown (see [12]) that this is essentially best possible by exhibiting a (discrete) system for which (1.6) holds but

$$
\pi(x)-\operatorname{li}(x)=\Omega\left(x e^{-c^{\prime} \sqrt{\log x}}\right) \quad \text { for some } c^{\prime}>0 .^{5}
$$

\subsection{Fractal membranes and Beurling zeta functions}

It may be useful to close this introduction by briefly explaining the connections between the general theme of this paper and the (new) notion of a fractal membrane (or 'quantum fractal string') recently introduced semi-heuristically by the second author in [27] and under current rigorous investigation (by Lapidus and Nest) in $[29]^{6}$. Recall that a fractal string $\mathcal{L}=\left\{l_{j}\right\}_{j=1}^{\infty}$ (see e.g. 24], 25], 31], 32], 28], 17], 34] and [33], Chapter 1 or [27, $\S 3.1$ for more details and motivations) is an open subset of $\mathbb{R}, \Omega=\cup_{j=1}^{\infty} I_{j} \subset \mathbb{R}$, whose connected components (i.e., the open intervals $I_{j}$ ) have lengths $l_{j}$ such that $\sum_{j=1}^{\infty} l_{j}^{d}<\infty$ for some $d>0$. Without loss of generality, we may assume that $l_{j} \downarrow 0$ and $1>l_{1} \geq l_{2} \geq$ $\cdots$, where $\mathcal{L}=\left\{l_{j}\right\}_{j=1}^{\infty}$ is written according to multiplicity.

In the forthcoming book [27] - entitled In Search of the Riemann Zeros and building, in particular, upon [33] and [26] - the second author proposes a (physically motivated

\footnotetext{
${ }^{5}$ Here, $f(x)=\Omega(g(x))$ as $x \rightarrow \infty$ means that there exists $c>0$ such that $|f(x)| \geq c g(x)$ for some arbitrarily large $x$.

${ }^{6}$ We stress, however, that the present paper can be read entirely independently of [27] and does not require any background on the theory of fractal strings or of fractal membranes. It is primarily a contribution to the theory of Beurling primes and zeta functions.
} 
and noncommutative) geometric framework within which to 'quantize' fractal strings. Given a fractal string $\mathcal{L}$, the resulting noncommutative geometric object $\mathcal{T}=\mathcal{T}_{\mathcal{L}}$ coined a fractal membrane or quantized fractal string - can be thought of heuristically as an (adelic, noncommutative) infinite dimensional torus, with underlying 'circles' of radii $R_{j}=1 / \log l_{j}^{-1}(j=1,2, \ldots)$. Further, the spectral (or quantum) partition function ${ }^{7}$ of $\mathcal{T}=\mathcal{T}_{\mathcal{L}}$ is given by

$$
Z_{\mathcal{T}}(s):=\sum_{j=1}^{\infty} e^{-\lambda_{j} s}=\prod_{j=1}^{\infty}\left(1-l_{j}^{s}\right)^{-1} .
$$

(See especially, [27, Chapters 3 and 4.) In other words, the spectrum $\left\{\lambda_{j}\right\}_{j=1}^{\infty}$ of the fractal membrane $\mathcal{T}=\mathcal{T}_{\mathcal{L}}$ is discrete and is given by the logarithms of the g-integers $\mathcal{N}=\left\{n_{j}\right\}$ based on the g-prime system $\mathcal{P}=\left\{p_{j}\right\}$, with $p_{j}=l_{j}^{-1}(j=1,2, \ldots)^{8}$. With this notation, $R_{j}=1 / \log p_{j}$ and the spectral partition function of $\mathcal{T}$ coincides with the Beurling zeta function of $\mathcal{P}: Z_{\mathcal{T}}(s)=\zeta_{\mathcal{P}}(s)$. Indeed, by $(1.7), Z_{\mathcal{T}}(s)$ is naturally given by the Dirichlet series in $\left(1.3^{\prime}\right)$ and the Euler product in (1.3).

Conjecturally (see [27], §4.4), $Z_{\mathcal{T}}$ should satisfy a generalised functional equation, connecting the (completed) partition functions of $\mathcal{T}$ and of its 'dual fractal membrane' $\mathcal{T}^{*}$, at the points $s$ and $1-s$. In [27, Chapter 5, is also introduced a 'moduli space of fractal membranes' 9 which enables one to obtain a natural (noncommutative) flow of zeta functions (and of g-prime systems), along with the corresponding flow of zeros. Conjecturally, [27], §§5.4-5.5, this continuous-time 'dynamical deformation' of Beurling zeta functions and prime systems would provide a new way to understand the remarkable role played (within the broader class of Beurling-type zeta functions) by arithmetic (or number-theoretic) zeta functions, such as the Riemann zeta function, which necessarily satisfy a 'self-dual functional equation' (i.e., symbolically, $\mathcal{T}=\mathcal{T}^{*}$ ).

We mention in closing that in joint work (in preparation) of the second author and Ryszard Nest, a rigorous operator-algebraic and noncommutative geometric [6] construction of fractal membranes is provided (in [29] $)^{10}$ and that (in [30) partial results are obtained towards some of the conjectures alluded to in the previous paragraph. From the mathematical point of view, however, much of the program proposed in 27] remains to be thoroughly investigated.

Although, as was stressed above, they primarily contribute to the standard theory of Beurling zeta functions and prime systems, the new results obtained in the present paper may also contribute to the concrete development of the theory of fractal membranes.

\footnotetext{
${ }^{7}$ defined as the trace of the 'heat semigroup' associated with $\mathcal{T}$.

${ }^{8}$ Physically, the eigenvalues $\lambda_{j}$ represent the 'energy levels' or the (square root of) the (vibrational) frequencies of the fractal membrane $\mathcal{T}$.

${ }^{9}$ Formally, this is the noncommutative space obtained as the quotient of the set of all fractal membranes by the following equivalence relation: $\mathcal{T} \sim \mathcal{T}^{\prime}$ iff $\mathcal{P} \sim \mathcal{P}^{\prime}$ (i.e., $\exists j_{0}, j_{1}$ such that $p_{j_{0}+q}=p_{j_{1}+q}^{\prime}, \forall q \geq 1$ ). Note that this preserves the poles and the zeros (with real part $\neq 0$ ) of the associated (equivalence class of) partition functions.

${ }^{10}$ It follows from 29] that fractal membranes are truly (second) quantized fractal strings, as suggested in [27].
} 


\section{$\S 2$. Further connections between $\pi(x), N(x)$ and $\zeta(s)$}

\subsection{Connecting $\pi(x)$ and $\zeta(s)$}

Throughout this paper, we shall use the weighted counting function

$$
\psi_{\mathcal{P}}(x)=\sum_{p^{k} \leq x, k \in \mathbb{N}} \log p=\sum_{n \leq x, n \in \mathcal{N}} \Lambda(n) \cdot{ }^{11}
$$

This is the natural counterpart for a g-prime system $\mathcal{P}$ of the Chebyshev-von Mangoldt weighted prime power counting function. As before, we shall often drop the reference to $\mathcal{P}$ if no confusion is likely. In the following, we shall also write

$$
\phi(s)=-\frac{\zeta^{\prime}(s)}{\zeta(s)}=\sum_{n \in \mathcal{N}} \frac{\Lambda(n)}{n^{s}} .
$$

The counting functions $N(x)$ and $\psi(x)$ are related to $\zeta(s)$ and $\phi(s)$ via

$$
\zeta(s)=s \int_{1}^{\infty} \frac{N(x)}{x^{s+1}} d x \quad \text { and } \quad \phi(s)=s \int_{1}^{\infty} \frac{\psi(x)}{x^{s+1}} d x .
$$

As a result, it is often more convenient to work with $\psi(x)$, rather than $\pi(x)$. Note that for $\alpha \in\left[\frac{1}{2}, 1\right)$, the statements

$$
\pi(x)=\operatorname{li}(x)+O\left(x^{\alpha+\varepsilon}\right) \quad(\forall \varepsilon>0) \quad \text { and } \quad \psi(x)=x+O\left(x^{\alpha+\varepsilon}\right) \quad(\forall \varepsilon>0),
$$

are equivalent. For $\mathcal{N}=\mathbb{N}$, it is well-known that the above are equivalent to the absence of zeros of the Riemann zeta function in the region $\{s \in \mathbb{C}: \Re s>\alpha\}$.

We first show that this holds more generally for g-prime systems.

\section{Theorem 2.1}

Suppose that for some $\alpha \in[0,1)$, we have

$$
\psi(x)=x+O\left(x^{\alpha+\varepsilon}\right) \quad \text { for all } \varepsilon>0 .
$$

Then $\zeta(s)$ has an analytic continuation to the half-plane $\{s \in \mathbb{C}: \Re s>\alpha\}$ except for a simple (non-removable) pole at $s=1$ and $\zeta(s) \neq 0$ in this region.

Conversely, suppose that for some $\alpha \in[0,1), \zeta(s)$ has an analytic continuation to the half-plane $\{s \in \mathbb{C}: \Re s>\alpha\}$, except for a simple (non-removable) pole at $s=1$, and that $\zeta(s) \neq 0$ in this region. Further assume that $|\phi(\sigma+i t)|=O\left(|t|^{\varepsilon}\right)$ holds for all $\varepsilon>0$, uniformly for $\sigma \geq \alpha+\delta$ with any $\delta>0$. Then

$$
\psi(x)=x+O\left(x^{\alpha+\varepsilon}\right) \quad \text { for all } \varepsilon>0 .
$$

The proof is standard except that in the converse part, an extra subtlety arises due to the possible close proximity of g-integers.

\footnotetext{
${ }^{11}$ Here $\Lambda=\Lambda_{\mathcal{P}}$ denotes the (generalised) von Mangoldt function, defined for $n$ in the multiset $\mathcal{N}$ by $\Lambda(n)=\log p$ if $n=p^{m}$ for some $p \in \mathcal{P}$ and $m \in \mathbb{N}$, and $\Lambda(n)=0$ otherwise.
} 
Proof. By hypothesis, $\psi(x)=x+r(x)$ where $r(x)=O\left(x^{\alpha+\varepsilon}\right)$ for all $\varepsilon>0$. It follows that

$$
\phi(s)=s \int_{1}^{\infty} \frac{x+r(x)}{x^{s+1}} d x=\frac{s}{s-1}+s \int_{1}^{\infty} \frac{r(x)}{x^{s+1}} d x .
$$

The latter integral converges for $\Re s>\alpha$ and represents an analytic function in this halfplane. This provides the analytic continuation of $\phi(s)$ to $\{s \in \mathbb{C}: \Re s>\alpha\}$ except for a simple pole at $s=1$ with residue 1 . By standard complex analysis, it follows that $\zeta(s)$ has an analytic continuation to $\{s \in \mathbb{C}: \Re s>\alpha\} \backslash\{1\}$, except for a simple (non-removable) pole at 1. Moreover, it has no zeros in this region, for if it did, then $\phi(s)=\frac{\zeta^{\prime}(s)}{\zeta(s)}$ would have a singularity.

For the converse, note that the hypothesis implies that $\phi(s)=-\frac{\zeta^{\prime}(s)}{\zeta(s)}$ has an analytic continuation to $\{s \in \mathbb{C}: \Re s>\alpha\}$ except for a simple pole at $s=1$ with residue 1 .

Let $c>1, T, x>0$ such that $x \notin \mathcal{N}$. Then, for $n \in \mathcal{N}$,

$$
\frac{1}{2 \pi i} \int_{c-i T}^{c+i T}\left(\frac{x}{n}\right)^{s} \frac{d s}{s}=O\left(\frac{(x / n)^{c}}{T|\log x / n|}\right)+\left\{\begin{array}{ll}
1 & \text { if } n<x \\
0 & \text { if } n>x
\end{array},\right.
$$

where the implied constant is independent of $n$ and $x$. Multiply through by $\Lambda(n)$ and sum over all $n \in \mathcal{N}$. Thus for $x \notin \mathcal{N}$, we have

$$
\frac{1}{2 \pi i} \int_{c-i T}^{c+i T} \frac{\phi(s) x^{s}}{s} d s=\psi(x)+O\left(\frac{x^{c}}{T} \sum_{n \in \mathcal{N}} \frac{\Lambda(n)}{n^{c}|\log x / n|}\right) .
$$

For $n \leq \frac{x}{2}$ and $n \geq 2 x,|\log x / n| \geq \log 2$, so

$$
\begin{aligned}
\psi(x) & =\frac{1}{2 \pi i} \int_{c-i T}^{c+i T} \frac{\phi(s) x^{s}}{s} d s+O\left(\frac{x^{c}}{T} \sum_{n \in \mathcal{N}} \frac{\Lambda(n)}{n^{c}}\right)+O\left(\frac{x^{c}}{T} \sum_{\frac{x}{2}<n<2 x} \frac{\Lambda(n)}{n^{c}|\log x / n|}\right) \\
& =\frac{1}{2 \pi i} \int_{c-i T}^{c+i T} \frac{\phi(s) x^{s}}{s} d s+O\left(\frac{x^{c}}{T(c-1)}\right)+O\left(\frac{x \log x}{T} \sum_{\frac{x}{2}<n<2 x} \frac{1}{|n-x|}\right),
\end{aligned}
$$

since $\phi(c)=O\left(\frac{1}{c-1}\right)$ and $|\log x / n|=\left|\log \left(1+\frac{n-x}{x}\right)\right| \asymp \frac{|n-x|}{x}$ for $\frac{x}{2}<n<2 x$.

Now consider the integral on the right of (2.1). We can push the contour past the pole at $s=1$ to the line $\Re s=\sigma$ for any $\sigma>\alpha$. The residue at 1 is $x$. Hence

$$
\frac{1}{2 \pi i} \int_{c-i T}^{c+i T} \frac{\phi(s) x^{s}}{s} d s=x+\frac{1}{2 \pi i}\left(\int_{c-i T}^{\sigma-i T}+\int_{\sigma-i T}^{\sigma+i T}+\int_{\sigma+i T}^{c+i T}\right) \frac{\phi(s) x^{s}}{s} d s .
$$

We estimate these integrals in turn, using $\phi(s)=O\left(|t|^{\varepsilon}\right)$. We have

$$
\left|\frac{1}{2 \pi i} \int_{\sigma+i T}^{c+i T} \frac{\phi(s) x^{s}}{s} d s\right| \leq \frac{x^{c}}{2 \pi T} \int_{\sigma}^{c}|\phi(y+i T)| d y=O\left(x^{c} T^{-1+\varepsilon}\right),
$$

and similarly for $\int_{c-i T}^{\sigma-i T}$, while

$$
\left|\frac{1}{2 \pi i} \int_{\sigma-i T}^{\sigma+i T} \frac{\phi(s) x^{s}}{s} d s\right| \leq \frac{x^{\sigma}}{2 \pi} \int_{-T}^{T} \frac{|\phi(\sigma+i t)|}{\sqrt{\sigma^{2}+t^{2}}} d t=O\left(x^{\sigma} T^{\varepsilon}\right) .
$$


Now choose $c=1+\frac{1}{\log x}$. Then (2.1) gives

$$
\psi(x)=x+O\left(x T^{-1+\varepsilon}\right)+O\left(x^{\sigma} T^{\varepsilon}\right)+O\left(\frac{x \log x}{T}\right)+O\left(\frac{x \log x}{T} \sum_{\frac{x}{2}<n<2 x} \frac{1}{|n-x|}\right)
$$

for $x \notin \mathcal{N}$ and every $\varepsilon>0$. We need to bound the sum on the right but this is difficult in general as $x$ can be arbitrarily close to a g-integer. So let's suppose that $x$ is such that there are no g-integers $n$ with $|n-x|<\frac{1}{x^{2}}$; i.e. $\left(x-\frac{1}{x^{2}}, x+\frac{1}{x^{2}}\right) \cap \mathcal{N}=\emptyset$. Then

$$
\sum_{\frac{x}{2}<n<2 x} \frac{1}{|n-x|} \leq x^{2} \sum_{\frac{x}{2}<n<2 x} 1 \leq x^{2} N(2 x)=O\left(x^{3}\right) .
$$

Taking $T=x^{4},(2.2)$ gives $\psi(x)=x+O\left(x^{\sigma+\varepsilon}\right)$ for all $\varepsilon>0$. This holds for all $\sigma>\alpha$ so

$$
\psi(x)=x+O\left(x^{\alpha+\varepsilon}\right)
$$

whenever $x \rightarrow \infty$ in such a way that $\left(x-\frac{1}{x^{2}}, x+\frac{1}{x^{2}}\right) \cap \mathcal{N}=\emptyset$.

Now we show that this is sufficient to prove the theorem. More precisely, we show the following: for all $x$ sufficiently large for which $\left(x-\frac{1}{x^{2}}, x+\frac{1}{x^{2}}\right) \cap \mathcal{N} \neq \emptyset, \exists x_{1} \in(x-3, x)$ and $x_{2} \in(x, x+3)$ such that

$$
\left(x_{1}-\frac{1}{x_{1}^{2}}, x_{1}+\frac{1}{x_{1}^{2}}\right) \cap \mathcal{N}=\emptyset \quad \text { and } \quad\left(x_{2}-\frac{1}{x_{2}^{2}}, x_{2}+\frac{1}{x_{2}^{2}}\right) \cap \mathcal{N}=\emptyset .
$$

Then the result will follow since $x=x_{r}+O(1)$ and $\psi\left(x_{r}\right)=x_{r}+O\left(x_{r}^{\alpha+\varepsilon}\right)$ (for $\left.r=1,2\right)$, so that

$$
\psi(x) \leq \psi\left(x_{2}\right)=x_{2}+O\left(x_{2}^{\alpha+\varepsilon}\right)=x+O\left(x^{\alpha+\varepsilon}\right)
$$

and

$$
\psi(x) \geq \psi\left(x_{1}\right)=x_{1}+O\left(x_{1}^{\alpha+\varepsilon}\right)=x+O\left(x^{\alpha+\varepsilon}\right) .
$$

It remains to prove $(2.3)$.

Suppose, for a contradiction, that there is no such $x_{2}$. Let $y_{n}=\sqrt[3]{x^{3}+9 n}$, for $n \in \mathbb{N}$. Thus each interval $\left(y_{n}-\frac{1}{y_{n}^{2}}, y_{n}+\frac{1}{y_{n}^{2}}\right)$ contains an element of $\mathcal{N}$ whenever $y_{n}<x+3$; i.e. for $n<x^{2}+3 x+3$. It is elementary to show that

$$
y_{n}+\frac{1}{y_{n}^{2}}<y_{n+1}-\frac{1}{y_{n+1}^{2}}
$$

so that these intervals are non-overlapping. This means that $N(x+3)-N(x) \geq x^{2}$. But this is false for all $x$ sufficiently large, as $N(x)=O(x)$.

The existence of $x_{1}$ is shown in a similar way using the sequence $z_{n}=\sqrt[3]{x^{3}-9 n}$, leading to $N(x)-N(x-3) \geq x^{2}$.

Note that if we had assumed the weaker bound $|\phi(\sigma+i t)|=O(|t|)$ for $\sigma>\alpha$, then one would only obtain

$$
\psi(x)=x+O\left(x^{\frac{\alpha+1}{2}+\varepsilon}\right) \quad \text { for all } \varepsilon>0
$$


2.2 Connections between $N(x)$ and $\psi(x)$

In the following, we consider the effect that the assumption on $\psi(x)$ of Theorem 2.1 has on the asymptotic behaviour of $N(x)$. This extends Diamond's result [9] (relating (1.5) to (1.4)) to the case $\beta=1$. It would be of interest to know if (apart from an improved value of $c$ ) this is essentially best possible.

\section{Theorem 2.2}

Suppose that $\psi(x)=x+O\left(x^{\alpha}\right)$ for some $\alpha \in(0,1)$. Then there exist positive constants $\rho$ and $c$ such that

$$
N(x)=\rho x+O\left(x e^{-c \sqrt{\log x \log \log x}}\right) .
$$

Proof. Let $\psi(x)=x+r(x)$, so that $r(x)=O\left(x^{\alpha}\right)$. We have already seen in the proof of Theorem 2.1 that this assumption implies the analytic continuation of $\phi(s)$ to $\Re s>\alpha$ except for a simple pole at $s=1$ with residue 1 , and that for $\Re s>\alpha$,

$$
s \int_{1}^{\infty} \frac{r(x)}{x^{s+1}} d x=\phi(s)-\frac{s}{s-1} .
$$

Now consider the sum $\sum_{n \leq x} \frac{\Lambda(n)}{n^{s}}$ for $\Re s>\alpha$, where $n$ ranges over elements of $\mathcal{N}$. We have

$$
\begin{aligned}
\sum_{n \leq x} \frac{\Lambda(n)}{n^{s}} & =\int_{1}^{x} \frac{d \psi(y)}{y^{s}}=\frac{\psi(x)}{x^{s}}+s \int_{1}^{x} \frac{\psi(y)}{y^{s+1}} d y \\
& =x^{1-s}+\frac{r(x)}{x^{s}}+s \int_{1}^{x} \frac{1}{y^{s}} d x+s \int_{1}^{x} \frac{r(y)}{y^{s+1}} d y \\
& =\frac{x^{1-s}}{1-s}+\phi(s)+\frac{r(x)}{x^{s}}-s \int_{x}^{\infty} \frac{r(y)}{y^{s+1}} d y
\end{aligned}
$$

Thus

$$
\phi(s)=\sum_{n \leq x} \frac{\Lambda(n)}{n^{s}}-\frac{x^{1-s}}{1-s}-\frac{r(x)}{x^{s}}+s \int_{x}^{\infty} \frac{r(y)}{y^{s+1}} d y .
$$

Writing $s=\sigma+i t$, and using $r(x)=O\left(x^{\alpha}\right)$, we obtain

$$
|\phi(\sigma+i t)| \leq \sum_{n \leq x} \frac{\Lambda(n)}{n^{\sigma}}+O\left(\frac{x^{1-\sigma}}{|t|}\right)+O\left(|t| x^{\alpha-\sigma}\right) .
$$

To estimate the first term on the right, put $t=0$ in (2.4) to give

$$
\begin{aligned}
\sum_{n \leq x} \frac{\Lambda(n)}{n^{\sigma}} & =\frac{x+r(x)}{x^{\sigma}}+\sigma \int_{1}^{x} y^{-\sigma} d y+\sigma \int_{1}^{x} \frac{r(y)}{y^{\sigma+1}} d y \\
& =x^{1-\sigma}+\frac{\sigma}{1-\sigma}\left(x^{1-\sigma}-1\right)+O\left(x^{\alpha-\sigma}\right)+O(1) \\
& =\frac{x^{1-\sigma}-1}{1-\sigma}+O(1) .
\end{aligned}
$$


This also holds for $\sigma=1$, if we interpret the first term on the right as $\log x$. Moreover, with this interpretation, the above estimate is uniform for $\sigma \in[\alpha+\delta, c]$ for any $c>1$ and $\delta>0$. Combining these gives

$$
|\phi(\sigma+i t)| \leq \frac{x^{1-\sigma}-1}{1-\sigma}+O(1)+O\left(\frac{x^{1-\sigma}}{|t|}\right)+O\left(|t| x^{\alpha-\sigma}\right) .
$$

The optimal choice for $x$ occurs when $x^{1-\sigma}$ and $|t| x^{\alpha-\sigma}$ are of the same order. So putting $x=|t|^{\frac{1}{1-\alpha}}$, we obtain

$$
|\phi(\sigma+i t)| \leq \frac{|t|^{\frac{1-\sigma}{1-\alpha}}-1}{1-\sigma}+O(1)+O\left(|t|^{\frac{1-\sigma}{1-\alpha}}\right) .
$$

Note that for $\sigma=1$ this is

$$
|\phi(1+i t)| \leq \frac{1}{1-\alpha} \log |t|+O(1)
$$

Now we use these inequalities to obtain bounds for $|\zeta(s)|$. For $\sigma \in(\alpha, 1)$,

$$
\begin{aligned}
\log \zeta(\sigma+i t) & =\int_{[\sigma+i t, 2+i t]} \phi(z) d z+\log \zeta(2+i t) \\
& =\int_{\sigma}^{2} \phi(y+i t) d y+O(1)
\end{aligned}
$$

Taking real parts, we obtain

$$
\log |\zeta(\sigma+i t)| \leq \int_{\sigma}^{2}|\phi(y+i t)| d y+O(1)
$$

Let $|t| \geq 3$ and put $\varepsilon(t)=(1-\alpha) \frac{\log \log |t|}{\log |t|}$. Then $\alpha<1-\varepsilon(t)<1$. Letting $\sigma=1-\varepsilon(t)$, we deduce from (2.5) that

$$
\log |\zeta(1-\varepsilon(t)+i t)| \leq \int_{1-\varepsilon(t)}^{2} \frac{|t|^{\frac{1-y}{1-\alpha}}-1}{1-y} d y+O(1)+O\left(\int_{1-\varepsilon(t)}^{2}|t|^{\frac{1-y}{1-\alpha}} d y\right)
$$

The latter integral equals

Hence

$$
\frac{1-\alpha}{\log |t|}\left(|t|^{\frac{\varepsilon(t)}{1-\alpha}}-|t|^{-\frac{1}{1-\alpha}}\right)<\frac{1-\alpha}{\log |t|^{\frac{\varepsilon(t)}{1-\alpha}}}=1-\alpha .
$$

$$
\begin{aligned}
\log |\zeta(1-\varepsilon(t)+i t)| & \leq \int_{1-\varepsilon(t)}^{2} \frac{|t|^{\frac{1-y}{1-\alpha}}-1}{1-y} d y+O(1) \\
& =\int_{0}^{\varepsilon(t)} \frac{|t|^{\frac{u}{1-\alpha}}-1}{u} d u+\int_{0}^{1} \frac{1-|t|^{-\frac{v}{1-\alpha}}}{v} d v+O(1) \\
& =\int_{0}^{\frac{\varepsilon(t) \log |t|}{1-\alpha}} \frac{e^{y}-1}{y} d y+\int_{0}^{\frac{\log |t|}{1-\alpha}} \frac{1-e^{-x}}{x} d x+O(1) \\
& =\int_{1}^{\log \log |t|} \frac{e^{y}}{y} d y+O(\log \log |t|) \\
& \sim \frac{\log |t|}{\log \log |t|} .
\end{aligned}
$$


Hence,

$$
|\zeta(1-\varepsilon(t)+i t)| \leq \exp \left(\frac{2 \log |t|}{\log \log |t|}\right),
$$

for all $|t|$ sufficiently large. We use this bound to find an approximate formula for $N_{1}(x)=$ $\int_{0}^{x} N(y) d y$, via the formula

$$
N_{1}(x)=\frac{1}{2 \pi i} \int_{c-i \infty}^{c+i \infty} \frac{\zeta(s)}{s(s+1)} x^{s+1} d s,
$$

which holds for any $c>1$. Pushing the contour past the simple pole at 1 gives

$$
N_{1}(x)=\frac{\rho}{2} x^{2}+\frac{1}{2 \pi i} \int_{\gamma} \frac{\zeta(s)}{s(s+1)} x^{s+1} d s,
$$

where $\gamma$ is the contour $s=1-\varepsilon(t)+i t$ for $|t| \geq 3$ and $s=1-\varepsilon(3)+i t$ for $|t| \leq 3$. This is allowed since

$$
\left|\int_{[c+i T, 1-\varepsilon(T)+i T]} \frac{\zeta(s)}{s(s+1)} x^{s+1} d s\right|=O\left(\frac{x^{c+1}}{T}\right) \rightarrow 0 \quad \text { as } T \rightarrow \infty .
$$

Now using the bound (2.6) for $|\zeta(s)|$ on $\gamma$, we obtain

$$
\begin{aligned}
\left|N_{1}(x)-\frac{\rho}{2} x^{2}\right| & =O\left(\int_{3}^{\infty} \frac{|\zeta(1-\varepsilon(t)+i t)|}{t^{2}} x^{2-\varepsilon(t)} d t\right)+O\left(x^{2-\varepsilon(3)}\right) \\
& =O\left(x^{2} \int_{3}^{\infty} \exp \left\{\frac{2 \log t}{\log \log t}-2 \log t-(1-\alpha) \frac{\log \log t \log x}{\log t}\right\} d t\right)+O\left(x^{2-\varepsilon(3)}\right) \\
& =O\left(x^{2} \int_{3}^{\infty} \exp \left\{-\frac{1}{2} u-(1-\alpha) \frac{\log u \log x}{u}\right\} d u\right)+O\left(x^{2-\varepsilon(3)}\right)
\end{aligned}
$$

Let $\lambda>0$ and split up the integral into two parts with ranges $[3, \lambda \sqrt{\log x \log \log x}]$ and $[\lambda \sqrt{\log x \log \log x}, \infty)$. For $u \leq \lambda \sqrt{\log x \log \log x}$, we have

$$
\frac{\log u \log x}{u} \geq \frac{1}{2 \lambda} \sqrt{\log x \log \log x},
$$

since $\log u / u$ decreases with $u$ for $u \geq e$. Hence

$$
\begin{aligned}
\int_{3}^{\infty} & \exp \left\{-\frac{1}{2} u-(1-\alpha) \frac{\log u \log x}{u}\right\} d u \\
& \leq e^{-\frac{1-\alpha}{2 \lambda} \sqrt{\log x \log \log x}} \int_{3}^{\lambda \sqrt{\log x \log \log x}} e^{-\frac{u}{2}} d u+\int_{\lambda \sqrt{\log x \log \log x}}^{\infty} e^{-\frac{u}{2}} d u \\
& =O\left(e^{-\frac{1-\alpha}{2 \lambda} \sqrt{\log x \log \log x}}\right)+O\left(e^{-\frac{\lambda}{2} \sqrt{\log x \log \log x}}\right) \\
& =O\left(e^{-\lambda^{\prime} \sqrt{\log x \log \log x}}\right),
\end{aligned}
$$

for some $\lambda^{\prime}>0$. In fact, the optimal choice is obtained by taking $\lambda$ such that $\lambda=\frac{1-\alpha}{\lambda}$; i.e. $\lambda=\sqrt{1-\alpha}$, which gives $\lambda^{\prime}=\frac{1}{2} \sqrt{1-\alpha}$. Hence (2.7) becomes

$$
N_{1}(x)=\frac{\rho}{2} x^{2}+O\left(x^{2} e^{-\frac{1}{2} \sqrt{(1-\alpha) \log x \log \log x}}\right) .
$$


By standard methods (using the fact that $N(x)$ increases with $x$ ), this yields

$$
N(x)=\rho x+O\left(x e^{-\frac{1}{4} \sqrt{(1-\alpha) \log x \log \log x}}\right) .
$$

\subsection{Well-behaved systems}

From the comments at the end of $\S 1.2$, we have seen that $N(x)=\rho x+O\left(x^{\theta}\right)$ for some $\theta<1$ does not imply that $\psi(x)=x+O\left(x^{\theta^{\prime}}\right)$ for some $\theta^{\prime}<1$. Nor does the converse seem to hold as Theorem 2.2 indicates. This suggests that we investigate g-prime systems where the functions $N(x)$ and $\psi(x)$ are simultaneously 'well-behaved'; that is, for some $\alpha<1$,

$$
\psi(x)=x+O\left(x^{\alpha}\right) \quad \text { and } \quad N(x)=\rho x+O\left(x^{\alpha}\right) .
$$

More precisely, for $0 \leq \alpha, \beta<1$, we define an $[\alpha, \beta]$-system to be a g-prime system for which

$$
\begin{aligned}
\psi(x) & =x+O\left(x^{\alpha+\varepsilon}\right) \\
N(x) & =\rho x+O\left(x^{\beta+\varepsilon}\right) \quad(\text { for some } \rho>0)
\end{aligned}
$$

hold for all $\varepsilon>0$, but for no $\varepsilon<0$.

\section{Conditional Examples}

(a) For $\mathbb{N}$, (2.9) holds with $\beta=0$ and if the Riemann Hypothesis were true, (2.8) would hold for $\alpha=\frac{1}{2}$ and this would show the existence of a $\left[\frac{1}{2}, 0\right]$-system.

(b) For the Gaussian integers of the field $\mathbb{Q}(i)$, the Dedekind zeta function is given by

$$
\frac{1}{1-2^{-s}} \prod_{p}\left(\frac{1}{1-p^{-s}}\right)^{2} \prod_{q}\left(\frac{1}{1-q^{-2 s}}\right)=\frac{1}{4} \sum_{n=1}^{\infty} \frac{r(n)}{n^{s}}
$$

where $p$ and $q$ run over the rational primes $1(\bmod 4)$ and $3(\bmod 4)$ respectively, and $r(n)$ is the number of ways of writing $n$ as $a^{2}+b^{2}$ with $a, b \in \mathbb{Z}$. The corresponding prime system $\mathcal{P}$ therefore consists of 2 , the rational primes $p \equiv 1(\bmod 4)$ occurring with multiplicity two, and the squares of the primes of the form $3(\bmod 4)$. Thus

$$
\pi_{\mathcal{P}}(x)=1+2 \pi_{1,4}(x)+\pi_{3,4}(\sqrt{x}),
$$

where $\pi_{k, m}(x)$ is the number of primes less than or equal to $x$ of the form $k(\bmod$ $m$ ). On the Generalised Riemann Hypothesis, one has

$$
\pi_{\mathcal{P}}(x)=\operatorname{li}(x)+O\left(x^{\frac{1}{2}+\varepsilon}\right) \text { for all } \varepsilon>0 .
$$

On the other hand, it is known that (see [19])

$$
N_{\mathcal{P}}(x)=\frac{1}{4} \sum_{n \leq x} r(n)=\frac{\pi}{4} x+O\left(x^{\frac{23}{73}}\right)
$$


and it is conjectured that the exponent in the error is actually $\frac{1}{4}+\varepsilon$ for all $\varepsilon>0$. Hence, assuming these conjectures, $\mathcal{P}$ is an example of a $\left[\frac{1}{2}, \frac{1}{4}\right]$-system.

From Theorem 2.1 we see that $(2.8)$ is equivalent to $\zeta(s)$ having a simple pole at $s=1$ and having no zeros in some vertical strip to the left of the line $\Re s=1$. Condition (2.9) is equivalent to $\zeta(s)$ being of finite order in such a strip; that is, there exists a positive constant $A$ such that

$$
\zeta(\sigma+i t)=O\left(|t|^{A}\right) \quad \text { as }|t| \rightarrow \infty \text { for } \sigma>\beta .
$$

However, if both (2.8) and (2.9) hold, then the bounds on $\zeta(\sigma+i t)$ are much stronger, in much the same way as the Riemann Hypothesis implies the Lindelöf Hypothesis.

\section{Theorem 2.3}

Let $\mathcal{P}$ be a $[\alpha, \beta]$-system. Then for $\sigma>\Theta=\max \{\alpha, \beta\}$, and uniformly for $\sigma \geq \Theta+\delta$ $($ any $\delta>0)$,

$$
\phi(\sigma+i t)=O\left((\log |t|)^{\frac{1-\sigma}{1-\Theta}+\varepsilon}\right) \quad \text { and } \quad \zeta(\sigma+i t)=O\left(\exp \left\{(\log |t|)^{\frac{1-\sigma}{1-\Theta}+\varepsilon}\right\}\right),
$$

for all $\varepsilon>0$. In particular, $\zeta(\sigma+i t)=O\left(|t|^{\varepsilon}\right)$ for all $\varepsilon>0$.

Proof. First, $\zeta(s)$ and $\phi(s)$ have analytic continuations to $\{s \in \mathbb{C}: \Re s>\min \{\alpha, \beta\}\} \backslash\{1\}$ with simple poles at $s=1$. Let $s=\sigma+i t$, with $\sigma>\min \{\alpha, \beta\}$. Then

$$
\begin{array}{ll}
\zeta(\sigma+i t)=O(|t|) & \text { for } \sigma>\beta, \text { and } \\
\phi(\sigma+i t)=O(|t|) & \text { for } \sigma>\alpha .
\end{array}
$$

Note that for any $\delta>0$, these hold uniformly as $|t| \rightarrow \infty$ for $\sigma \geq \beta+\delta$, and $\sigma \geq \alpha+\delta$ respectively.

By Theorem 2.1, $\zeta(s)$ is non-zero for $\Re s>\alpha$, and so $\log \zeta(s)$ exists and is analytic on $\{s \in \mathbb{C}: \Re s>\alpha\} \backslash(\alpha, 1]$. Hence for $\sigma>\Theta=\max \{\alpha, \beta\}$, and uniformly for $\sigma \geq \Theta+\delta$, we have

$$
\Re \log \zeta(\sigma+i t)=\log |\zeta(\sigma+i t)| \leq A \log |t|,
$$

for some $A$. Applying the Borel-Carathéodory Theorem (see 40]), it follows that $\mid \log \zeta(\sigma+$ $i t) \mid=O(\log |t|)$ uniformly for $\sigma \geq \Theta+2 \delta$. Now by Cauchy's Theorem,

$$
\phi(\sigma+i t)=-\frac{1}{2 \pi i} \int_{\gamma} \frac{\log \zeta(z)}{(z-\sigma-i t)^{2}} d z,
$$

where $\gamma$ is the circle with centre $\sigma+i$ and radius $\varepsilon$. Choosing $\varepsilon>0$ so that $\sigma-\varepsilon>\Theta$, gives

$$
|\phi(\sigma+i t)| \leq \frac{1}{\varepsilon} \sup _{z \in \gamma}|\log \zeta(z)|=O(\log |t|) .
$$

Let $\delta, \eta>0$. We apply Hadamard's Three-Circles Theorem to the circles $C_{1}, C_{2}, C_{3}$ with centre $c+i t(c>1+\eta)$, passing through the points $1+\eta+i t, \sigma+i t, \Theta+\delta+i t$ respectively. 
The radii are $c-1-\eta, c-\sigma, c-\Theta-\delta$ respectively. Let $M_{1}, M_{2}, M_{3}$ be the maxima of $|\phi(s)|$ on each of the circles $C_{1}, C_{2}, C_{3}$. Then,

$$
M_{2} \leq M_{1}^{1-\kappa} M_{3}^{\kappa}, \quad \text { where } \quad \kappa=\frac{\log \left(\frac{c-\sigma}{c-1-\eta}\right)}{\log \left(\frac{c-\Theta-\delta}{c-1-\eta}\right)} .
$$

Now $M_{3}=O(\log |t|)$ by estimate (2.10), and

$$
M_{1}=\max _{z \in C_{1}}|\phi(z)| \leq \max _{z \in C_{1}} \phi(\Re z) \leq \phi(1+\eta)=O(1) .
$$

Hence $M_{2}=O\left((\log |t|)^{\kappa}\right)$, and in particular,

$$
|\phi(\sigma+i t)|=O\left((\log |t|)^{\kappa}\right)
$$

The exponent, $\kappa$, can be made as close as we please to $\frac{1-\sigma}{1-\Theta}$ by choosing $c$ large and $\eta, \delta$ small, since

$$
\begin{aligned}
\kappa & =\frac{\log \left(\frac{c-\sigma}{c-1-\eta}\right)}{\log \left(\frac{c-\Theta-\delta}{c-1-\eta}\right)}=\frac{\log \left(\frac{1-\sigma / c}{1-(1+\eta) / c}\right)}{\log \left(\frac{1-(\Theta+\delta) / c}{1-(1+\eta) / c}\right)}=\frac{1+\eta-\sigma+O\left(\frac{1}{c}\right)}{1+\eta-(\Theta+\delta)+O\left(\frac{1}{c}\right)} \\
& =\frac{1-\sigma}{1-\Theta}+O(\eta)+O(\delta)+O(1 / c) .
\end{aligned}
$$

Hence

$$
|\phi(\sigma+i t)|=O\left((\log |t|)^{\frac{1-\sigma}{1-\Theta}+\varepsilon}\right) \quad \text { for any } \varepsilon>0
$$

Finally,

$$
\begin{aligned}
\log |\zeta(\sigma+i t)| & =\Re\left\{\int_{[\sigma+i t, 2+i t]} \phi(z) d z\right\}+\log |\zeta(2+i t)| \leq \int_{\sigma}^{2}|\phi(x+i t)| d x+A \\
& =O\left(\int_{\sigma}^{2}(\log |t|)^{\frac{1-x}{1-\Theta}+\varepsilon} d x\right)=O\left((\log |t|)^{\frac{1-\sigma}{1-\Theta}+\varepsilon}\right)
\end{aligned}
$$

so that $|\zeta(\sigma+i t)|=O\left(\exp \left((\log |t|)^{\frac{1-\sigma}{1-\Theta}+\varepsilon}\right)\right)$. Since the exponent is less than one for $\sigma>\Theta$ (by taking $\varepsilon$ sufficiently small), it follows that $|\zeta(\sigma+i t)|=O\left(|t|^{\varepsilon}\right)$ for all $\varepsilon>0$.

Remark (i) If $\alpha<\beta$ and we already know that $\zeta(s)$ is of finite order for $\sigma>\eta$ for some $\eta \in(\alpha, \beta)$, then $\zeta(s)$ and $\phi(s)$ have zero order in this range.

(ii) If $\beta<\alpha$ and we already know that $\phi(s)$ has only finitely many poles for $\sigma>\eta^{\prime}$ (equivalently, $\zeta(s)$ has finitely many zeros here), then $\zeta(s)$ and $\phi(s)$ have zero order in this range.

For functions $f$ of finite order we define, as usual, the $\operatorname{order} \mu_{f}(\sigma)$ to be the infimum of all real numbers $\lambda$ such that

$$
f(\sigma+i t)=O\left(|t|^{\lambda}\right) \quad \text { as }|t| \rightarrow \infty
$$


If there is no such $\lambda$, we shall write $\mu_{f}(\sigma)=\infty$ and say that $f$ is of infinite order. It is well-known that, as a function of $\sigma, \mu_{f}(\sigma)$ is non-negative, decreasing, and convex. Theorem 2.3 tells us that $\mu_{\zeta}(\sigma)=0$ for $\sigma>\Theta$.

We are naturally led to consider the cases where $\alpha=\beta, \alpha>\beta$, and $\alpha<\beta$. The case $\alpha=\beta$ just tells us that $\zeta(s)$ has no zeros and is of zero order for $\Re s>\alpha$. Of course, we can say nothing about what happens for $\Re s \leq \alpha$. This leaves us with the more interesting possibilities:

Case A: $\alpha>\beta$.

This looks very similar to the case of $\mathbb{N}$ under the assumption of the Riemann Hypothesis. In the half-plane where $\Re s=\sigma>\alpha, \mu_{\zeta}(\sigma)=0$ and $\zeta(s)$ has no zeros, while in the strip $\beta<\sigma \leq \alpha$, we have $\mu_{\zeta}(\sigma)<1$ and there must be infinitely many zeros on, or arbitrarily close to, the line $\Re s=\alpha$. (If not, then, after Remark (ii), in a strip to the left of $\Re s=\alpha$, $\zeta(s)$ would be non-zero and of zero order, leading to $\psi(x)=x+O\left(x^{\lambda}\right)$ for some $\lambda<\alpha$ using Theorem 2.1, contradicting the minimality of $\alpha$.) See Case A of Figure 1 for a summary of this discussion.

Case B: $\alpha<\beta$.

This case is quite different. Now $\zeta(s)$ has no zeros for $\Re s>\alpha$, has zero order for $\Re s>\beta$, and must be of infinite order in the strip $\alpha<\Re s<\beta$. For if $\zeta(s)$ is of finite order in some strip to the left of $\beta$, then, by Remark (i), $\mu_{\zeta}(\sigma)=0$ in such a strip. Hence either the order is zero or infinite for $\alpha<\Re s<\beta$. However, we can rule out the case of zero order since it would imply from (a slightly adjusted) Theorem 2.1 that $N(x)=x+O\left(x^{\lambda}\right)$ for some $\lambda<\beta$, contradicting the minimality of $\beta$. See Case B of Figure 1.

All the naturally occurring examples of generalised prime systems, such as those arising from the Dedekind zeta function, or more generally, from the Selberg class (39] and e.g. [7], 37]) of zeta functions (with a standard Euler product), possess a zeta function of finite order. Thus assuming the Generalised Riemann Hypothesis, or Selberg's conjecture regarding the zeros of the corresponding zeta function, we find that all these are examples of systems $\left[\frac{1}{2}, \beta\right]$ with $\beta \leq \frac{1}{2}$. In fact, it may reasonably be conjectured that these are actually systems $\left[\frac{1}{2}, \frac{1}{2}-\frac{1}{2 d}\right]$, where $d$ is the degree of the field extension, or more generally, the degree of the Selberg zeta function.

\section{$\S 3$. Analytic continuation and Functional equation}

\subsection{Analytic continuation}

The classical Riemann zeta function can be continued analytically to the whole plane except for a simple pole at $s=1$, so it is natural to ask if, or under what conditions, this occurs for $\zeta(s)=\zeta_{\mathcal{P}}(s)$, the Beurling zeta function associated to a prime system $\mathcal{P}$. In general, one would expect the line $\Re s=1$ to be a natural boundary.

We have already seen (in §2) that an analytic continuation exists if either of

$$
N(x)=\rho x+O\left(x^{\alpha}\right), \quad \psi(x)=x+O\left(x^{\alpha}\right)
$$


no

zeros zeros
0
$\beta$
$\alpha$
1
$\mu<1 \quad \mu=0$

$\alpha \quad \beta$
$\mu=\infty \quad \mu=0$

Case $A$ no zeros

Case $B$

Figure 1: Cases $\mathrm{A}(\alpha>\beta)$ and $\mathrm{B}(\alpha<\beta)$ for well-behaved systems

holds for some $\alpha<1$. One problem with such an approach is that there is no hope of extending $\zeta(s)$ beyond $\Re s=0$ since $\alpha \geq 0$ in any case.

There are other methods for obtaining analytic continuations. One way is to consider the function defined for $\Re z>0$ by the series (akin to a 'partition function')

$$
F(z)=\sum_{n \in \mathcal{N}} e^{-n z}
$$

Then

$$
\Gamma(s) \zeta(s)=\sum_{n \in \mathcal{N}} \int_{0}^{\infty} x^{s-1} e^{-n x} d x=\int_{0}^{\infty} x^{s-1} F(x) d x \quad \text { for } \Re s>1 .
$$

The integral $\int_{1}^{\infty} x^{s-1} F(x) d x$ extends to an entire function since $F(x)=O\left(e^{-x}\right)$, while knowing the behaviour of $F(x)$ near $x=0$ can lead to an extension for $\int_{0}^{1} x^{s-1} F(x) d x$.

Indeed, this method may be generalised by using kernels other than $e^{-x}$; that is, let

$$
F(x)=\sum_{n \in \mathcal{N}} g(n x)
$$

for some function $g(x)$ defined on the positive reals with suitable behaviour at infinity. By defining the corresponding 'gamma' function via the Mellin transform

$$
G(s)=\int_{0}^{\infty} x^{s-1} g(x) d x
$$


one finds (formally) that

$$
G(s) \zeta(s)=\int_{0}^{\infty} x^{s-1} F(x) d x .
$$

We shall suppose $F(x)$ has an asymptotic expansion as $x \rightarrow 0^{+}$of the form

$$
F(x) \approx \sum_{n=1}^{\infty} \frac{P_{n}(\log x)}{x^{\lambda_{n}}}
$$

where the $P_{n}(\cdot)$ are polynomials and $\lambda_{n}$ is a sequence of distinct complex numbers with real parts decreasing to minus infinity. Then we have the following proposition of which we include the proof for completeness.

\section{Proposition 3.1}

Let $g:(0, \infty) \rightarrow \mathbb{C}$ be a continuous function such that $g(x)=O\left(x^{\alpha}\right)$ as $x \rightarrow 0^{+}$and $g(x)=O\left(x^{-\beta}\right)$ as $x \rightarrow \infty$ for some $\alpha<1<\beta$. Let $F(x)$ and $G(s)$ be defined by (3.1) and (3.2) for $x>0$ and $\Re s \in(\alpha, \beta)$ respectively. Then

$$
G(s) \zeta(s)=\int_{0}^{\infty} x^{s-1} F(x) d x \quad \text { for } 1<\Re s<\beta .
$$

Furthermore, if $F(x)$ possesses an asymptotic expansion of the form (3.3) as $x \rightarrow 0^{+}$, then $G(s) \zeta(s)$ has a continuation to $\{s \in \mathbb{C}: \Re s<\beta\}$ which is analytic except for poles at each of the $\lambda_{n}$, the order of the pole being equal to the degree of $P_{n}(\cdot)$ plus 1.

Proof. The integral (3.2) for $G(s)$ converges absolutely and uniformly on compact subsets of the strip $\alpha<\Re s<\beta$ and $G(s)$ is analytic there, while the series (3.1) for $F(x)$ converges absolutely for $x>0$. Thus, we have for $\Re s \in(1, \beta)$,

$$
G(s) \zeta(s)=\sum_{n \in \mathcal{N}} \frac{1}{n^{s}} \int_{0}^{\infty} x^{s-1} g(x) d x=\sum_{n \in \mathcal{N}} \int_{0}^{\infty} x^{s-1} g(n x) d x=\int_{0}^{\infty} x^{s-1} F(x) d x .
$$

The last step of interchanging the integral and sum is justified because of the absolute convergence of the series. To obtain the analytic continuation, we write,

$$
G(s) \zeta(s)=\int_{0}^{1} x^{s-1} F(x) d x+\int_{1}^{\infty} x^{s-1} F(x) d x .
$$

The latter integral converges for $\Re s<\beta$ since $F(x) \leq \sum_{n \in \mathcal{N}} \frac{A}{(n x)^{\beta}}=O\left(x^{-\beta}\right)$, so we need only consider the former integral. By (3.3), we may write

$$
F(x)=F_{N}(x)+E_{N}(x),
$$

for each $N$ and some $k$, where

$$
F_{N}(x)=\sum_{n=1}^{N} \frac{P_{n}(\log x)}{x^{\lambda_{n}}} \quad \text { and } \quad E_{N}(x)=O\left(x^{-\lambda_{N}}(\log x)^{k}\right) .
$$


Thus

$$
\begin{aligned}
\int_{0}^{1} x^{s-1} F(x) d x & =\int_{0}^{1} x^{s-1} F_{N}(x) d x+\int_{0}^{1} x^{s-1} E_{N}(x) d x \\
& =\sum_{n=1}^{N} \int_{0}^{1} P_{n}(\log x) x^{s-1-\lambda_{n}} d x+h_{N}(s),
\end{aligned}
$$

where $h_{N}(s)$ is analytic for $\Re s>\Re \lambda_{N}$. But each of the integrals $\int_{0}^{1} P_{n}(\log x) x^{s-1-\lambda_{n}} d x$ is a polynomial in $\frac{1}{\lambda_{n}-s}$ since if $P_{n}(x)=a_{0}+a_{1} x+\ldots+a_{d} x^{d}$, then

$$
\int_{0}^{1} P_{n}(\log x) x^{s-1-\lambda_{n}} d x=\int_{0}^{\infty} P_{n}(-t) e^{-t\left(s-\lambda_{n}\right)} d t=-\sum_{m=0}^{d} \frac{m ! a_{m}}{\left(\lambda_{n}-s\right)^{m+1}} .
$$

Hence $\int_{0}^{1} x^{s-1} F(x) d x$ has an analytic continuation to $\Re s>\lambda_{N}$ except for poles at $\lambda_{1}, \ldots, \lambda_{N}$, whose order is the degree of $P_{n}(x)$ plus 1 . This holds for all integers $N \geq 1$, and since $\lambda_{N} \rightarrow-\infty$ as $N \rightarrow \infty$, we deduce that the meromorphic continuation of $G(s) \zeta(s)$ for $\Re s<\beta$.

\subsection{Functional Equation for $\zeta(s)$}

Riemann's $\zeta(s)$ satisfies the well-known functional equation

$$
\zeta(1-s)=2^{1-s} \pi^{-s} \cos \left(\frac{\pi s}{2}\right) \Gamma(s) \zeta(s)
$$

which may also be written in the form

$$
\pi^{-(1-s) / 2} \Gamma\left(\frac{1-s}{2}\right) \zeta(1-s)=\pi^{-s / 2} \Gamma\left(\frac{s}{2}\right) \zeta(s) .
$$

We can ask whether some form of functional equation still holds for more general Beurling zeta functions $\zeta_{\mathcal{P}}(s)$. Specifically, under what circumstances does an equation of the form

$$
G_{1}(1-s) \zeta_{1}(1-s)=G_{2}(s) \zeta_{2}(s),
$$

hold? Here the $G_{r}(s)$ are 'Gamma'-like functions, say defined by Mellin transforms of given functions $g_{r}(x)$ as in (3.2), and the $\zeta_{r}(s)$ are Euler products for two (possibly different) prime systems. It is well-known that (3.4) is equivalent to the modular identity:

$$
\sum_{-\infty}^{\infty} e^{-\pi n^{2} x^{2}}=\frac{1}{x} \sum_{-\infty}^{\infty} e^{-\pi n^{2} / x^{2}}
$$

It follows from Theorem 3.2 below that, under some mild conditions on the $g_{r}(x),(3.5)$ is also equivalent to an identity of this type. For similar equivalences in a related context, see [3].

\section{Theorem 3.2}

For $r=1,2$, let $F_{r}:(0, \infty) \rightarrow \mathbb{C}$ be continuous functions which are $O\left(x^{-1-\varepsilon}\right)$ as $x \rightarrow 0^{+}$ for every $\varepsilon>0$ and $O\left(x^{-\beta_{r}}\right)$ as $x \rightarrow \infty$, for some $\beta_{r}>1$. Let $\Psi_{r}(s)$ be the Mellin transform of $F_{r}(x)$ defined for $\Re s \in\left(1, \beta_{r}\right)$. Then the following are equivalent: 
(a) $\Psi_{1}(s)$ and $\Psi_{2}(s)$ have analytic continuations to $1-\beta_{2}<\Re s<\beta_{1}$ and $1-\beta_{1}<\Re s<$ $\beta_{2}$ respectively except for a finite number of poles in any given strip $\sigma_{1} \leq \Re s \leq \sigma_{2}$, tend to 0 uniformly as $|\Im s| \rightarrow \infty$, and satisfy the functional equation

$$
\Psi_{1}(1-s)=\Psi_{2}(s) \quad \text { for } 1-\beta_{1}<\Re s<\beta_{2} .
$$

(b)

$$
F_{1}(x)=\frac{1}{x} F_{2}\left(\frac{1}{x}\right)+H(x)
$$

where $H(x)$ is a finite series of the form $\sum_{k} a_{k} x^{\mu_{k}}(\log x)^{\nu_{k}}$ with $\mu_{k} \in \mathbb{C}$ and $\nu_{k} \in \mathbb{N}_{0}$.

For the specific form of functional equation (3.5), we take

$$
F_{r}(x)=\sum_{n \in \mathcal{N}_{r}} g_{r}(n x) \quad(r=1,2)
$$

where $\mathcal{N}_{r}$ are two g-integer systems, the $g_{r}(x)$ are as before and satisfy $O\left(x^{-\alpha_{r}}\right)$ and $O\left(x^{\beta_{r}}\right)$ as $x \rightarrow 0^{+}$and $x \rightarrow \infty$ respectively, for some $\alpha_{r}<1<\beta_{r}$. Then $F_{r}(x)$ has the appropriate behaviour at 0 and infinity, and $\Psi_{r}(s)=G_{r}(s) \zeta_{r}(s)$.

Addendum After the completion of this paper, it was pointed out to us that S. Bochner ([5], Theorems 2 and 3) had obtained a very similar result. There is a minor difference. In Bochner's case, the function $\Psi_{1}(s)$ is allowed to have infinitely many poles in a given strip rather than finitely many. This implies that $H(x)$ does not have the closed form that we obtain, but is a more general 'residual' function.

Proof of Theorem 3.2. (a) $\Longrightarrow(\mathrm{b})$. By the inverse Mellin transform for $F_{2}(x)$, we have

$$
F_{2}(x)=\frac{1}{2 \pi i} \int_{(c)} \Psi_{2}(s) x^{-s} d s \quad \text { for any } c \in\left(1, \beta_{2}\right),
$$

where $\int_{(c)}$ denotes $\lim _{T \rightarrow \infty} \int_{c-i T}^{c+i T}$. Hence

$$
\begin{aligned}
\frac{1}{x} F_{2}\left(\frac{1}{x}\right) & =\frac{1}{2 \pi i} \int_{(c)} \Psi_{2}(s) x^{s-1} d s \\
= & \frac{1}{2 \pi i} \int_{(c)} \Psi_{1}(1-s) x^{s-1} d s \\
= & \frac{1}{2 \pi i} \int_{\left(c^{\prime}\right)} \Psi_{1}(s) x^{-s} d s \quad\left(\text { where } c^{\prime}=1-c\right) .
\end{aligned}
$$

On the other hand,

$$
F_{1}(x)=\frac{1}{2 \pi i} \int_{(c)} \Psi_{1}(s) x^{-s} d s \quad \text { for any } c \in\left(1, \beta_{1}\right) .
$$

Moving the contour from the line $\Re s=c$ to $\Re s=c^{\prime}$, we pick up the residues at the poles of $\Psi_{1}(s)$ in the strip $\{s: 0 \leq \Re s \leq 1\}$. By assumption there are only a finite number of 
these and the residues of the integrand are all of the form $a x^{\mu} P(\log x)$ for some $a, \mu \in \mathbb{C}$ and some polynomial $P(\cdot)$. Moving the contour is permissible here by the assumption that $\Psi_{1}(\sigma+i T) \rightarrow 0$ uniformly as $|T| \rightarrow \infty$. Hence

$$
F_{1}(x)=\frac{1}{x} F_{2}\left(\frac{1}{x}\right)+\sum_{k} a_{k} x^{\mu_{k}}(\log x)^{\nu_{k}}
$$

for some constants $a_{k}, \mu_{k} \in \mathbb{C}$ and $\nu_{k} \in \mathbb{N}_{0}$.

(b) $\Longrightarrow(\mathrm{a})$. For $r=1,2$, we write

$$
\Psi_{r}(s)=\int_{0}^{1} x^{s-1} F_{r}(x) d x+\int_{1}^{\infty} x^{s-1} F_{r}(x) d x=A_{r}(s)+B_{r}(s),
$$

where $A_{r}(s)=\int_{0}^{1} x^{s-1} F_{r}(x) d x$ and $B_{r}(s)=\int_{1}^{\infty} x^{s-1} F_{r}(x) d x$. These integrals converge for $\Re s>1$ and $\Re s<\beta_{r}$ respectively. Now

$$
\begin{aligned}
A_{1}(s) & =\int_{0}^{1} x^{s-1} F_{1}(x) d x=\int_{0}^{1} x^{s-1}\left(\frac{1}{x} F_{2}\left(\frac{1}{x}\right)+H(x)\right) d x \\
& =\int_{1}^{\infty} x^{-s} F_{2}(x) d x+\int_{0}^{1} x^{s-1} H(x) d x \\
& =B_{2}(1-s)+H_{1}(s) .
\end{aligned}
$$

On the other hand,

$$
\begin{aligned}
B_{1}(s) & =\int_{1}^{\infty} x^{s-1} F_{1}(x) d x=\int_{1}^{\infty} x^{s-1}\left(\frac{1}{x} F_{2}\left(\frac{1}{x}\right)+H(x)\right) d x \\
& =\int_{0}^{1} x^{-s} F_{2}(x) d x+\int_{1}^{\infty} x^{s-1} H(x) d x \\
& =A_{2}(1-s)+H_{2}(s) .
\end{aligned}
$$

Where do (3.7) and (3.8) hold? The functions $H_{1}(s)$ and $H_{2}(s)$ are actually rational functions as shown below and hence have meromorphic continuations to $\mathbb{C}$. Now $A_{1}(s)$ is analytic for $\Re s>1$ while $B_{2}(1-s)$ is analytic for $\Re s>1-\beta_{2}$. Thus $A_{1}(s)$ has a meromorphic continuation for $\Re s>1-\beta_{2}$ and (3.7) holds for $\Re s>1-\beta_{2}$. Similarly, (3.8) holds for $\Re s<\beta_{1}$. These provide the required continuations of $\Psi_{1}(s)$ and $\Psi_{2}(s)$. In particular, for $1-\beta_{2}<\Re s<\beta_{1}$, (3.7) and (3.8) hold simultaneously and together give

$$
A_{1}(s)+B_{1}(s)=A_{2}(1-s)+B_{2}(1-s)+H_{1}(s)+H_{2}(s),
$$

i.e.

$$
\Psi_{1}(s)=\Psi_{2}(1-s)+H_{1}(s)+H_{2}(s) .
$$

We proceed to show that $H_{1}(s)$ and $H_{2}(s)$ are rational functions such that $H_{1}(s)+H_{2}(s)=$ 0 . Indeed, we have

$$
\begin{aligned}
H_{1}(s) & =\int_{0}^{1} x^{s-1} H(x) d x=\sum_{k} a_{k} \int_{0}^{1} x^{s+\mu_{k}-1}(\log x)^{\nu_{k}} d x \quad\left(x=e^{-t}\right) \\
& =\sum_{k} a_{k}(-1)^{\nu_{k}} \int_{0}^{\infty} t^{\nu_{k}} e^{-\left(s+\mu_{k}\right) t} d t
\end{aligned}
$$


the integral converging whenever $\Re\left(s+\mu_{k}\right)>0$ for all $k$. For $H_{2}(s)$ we have

$$
\begin{aligned}
H_{2}(s) & =\int_{1}^{\infty} x^{s-1} H(x) d x=\sum_{k} a_{k} \int_{1}^{\infty} x^{s+\mu_{k}-1}(\log x)^{\nu_{k}} d x \quad\left(x=e^{t}\right) \\
& =\sum_{k} a_{k} \int_{0}^{\infty} t^{\nu_{k}} e^{\left(s+\mu_{k}\right) t} d t
\end{aligned}
$$

the integral converging whenever $\Re\left(s+\mu_{k}\right)<0$ for all $k$. But for $m$ a non-negative integer and $\alpha \in \mathbb{C}$ with positive real part, $\int_{0}^{\infty} t^{m} e^{-\alpha t} d t=m ! \alpha^{-m-1}$. Thus

$$
\begin{gathered}
H_{1}(s)=\sum_{k} \frac{a_{k}(-1)^{\nu_{k}} \nu_{k} !}{\left(s+\mu_{k}\right)^{\nu_{k}+1}}, \quad \text { and } \\
H_{2}(s)=\sum_{k} \frac{a_{k} \nu_{k} !}{\left(-\left(s+\mu_{k}\right)\right)^{\nu_{k}+1}}=\sum_{k} \frac{a_{k}(-1)^{\nu_{k}+1} \nu_{k} !}{\left(s+\mu_{k}\right)^{\nu_{k}+1}}=-H_{1}(s),
\end{gathered}
$$

as required.

Finally, we show that $\left|\Psi_{r}(s)\right| \rightarrow 0$ uniformly as $|\Im s| \rightarrow \infty$, for $r=1,2$. By the functional equation, it suffices to show this for $\left|\Psi_{1}(s)\right|$. Let $s=\sigma+i t$. We have

$$
\Psi_{1}(s)=A_{1}(s)+B_{1}(s)=B_{2}(1-s)+B_{1}(s)+H_{1}(s) .
$$

Now $\left|H_{1}(s)\right|=O(1 /|t|)$, while

$$
\left|B_{1}(\sigma+i t)\right|=\left|\int_{1}^{\infty} x^{\sigma-1+i t} F_{1}(x) d x\right|=\left|\int_{1}^{\infty} x^{\sigma-1} F_{1}(x) e^{i t \log x} d x\right| .
$$

The integral $\int_{1}^{\infty} x^{\sigma-1} F_{1}(x) d x$ converges uniformly whenever $\sigma \leq \beta_{1}-\delta$ for any $\delta>0$; hence by the Riemann-Lebesgue Theorem, it follows that the RHS integral tends to 0 as $|t| \rightarrow \infty$ uniformly for $\sigma \leq \beta_{1}-\delta$. Similarly, $\left|B_{2}(1-\sigma+i t)\right| \rightarrow 0$ uniformly for $\sigma \geq 1-\beta_{2}+\delta$. Hence $\left|\Psi_{1}(\sigma+i t)\right| \rightarrow 0$ uniformly as $|t| \rightarrow \infty$ for $1-\beta_{2}+\delta \leq \sigma \leq \beta_{1}-\delta$.

Remark. In a work in preparation [30, the second author and R. Nest have obtained (under hypotheses similar to those of Proposition 3.1) a generalised functional equation for $\zeta(s)$. The latter connects a suitable completion of the Beurling zeta function $\zeta(s)$ associated to the g-prime system $\mathcal{P}$ to that of $\zeta^{*}(s)$, associated to a 'dual system' $\mathcal{P}^{*}$ (which involves in general a continuous g-prime system); ${ }^{12}$ this establishes part of a conjecture formulated in [27], §4.4. (see $\$ 1.3$ above). We note that the context of [30] is not restricted to Beurling zeta functions and applies, in particular, to zeta functions associated with quasicrystals.

\subsection{Functional Equation for $\zeta(s)$}

Riemann's $\zeta(s)$ satisfies the well-known functional equation

$$
\zeta(1-s)=2^{1-s} \pi^{-s} \cos \left(\frac{\pi s}{2}\right) \Gamma(s) \zeta(s)
$$

\footnotetext{
${ }^{12}$ Determining when these g-prime systems have an infinite discrete part is an interesting and difficult problem that is far from resolved at this stage.
} 
which may also be written in the form

$$
\pi^{-(1-s) / 2} \Gamma\left(\frac{1-s}{2}\right) \zeta(1-s)=\pi^{-s / 2} \Gamma\left(\frac{s}{2}\right) \zeta(s)
$$

We can ask whether some form of functional equation still holds for more general Beurling zeta functions $\zeta_{\mathcal{P}}(s)$. Specifically, under what circumstances does an equation of the form

$$
G_{1}(1-s) \zeta_{1}(1-s)=G_{2}(s) \zeta_{2}(s)
$$

hold? Here the $G_{r}(s)$ are 'Gamma'-like functions, say defined by Mellin transforms of given functions $g_{r}(x)$ as in (3.2), and the $\zeta_{r}(s)$ are Euler products for two (possibly different) prime systems. It is well-known that (3.4) is equivalent to the modular identity:

$$
\sum_{-\infty}^{\infty} e^{-\pi n^{2} x^{2}}=\frac{1}{x} \sum_{-\infty}^{\infty} e^{-\pi n^{2} / x^{2}}
$$

It follows from Theorem 3.2 below that, under some mild conditions on the $g_{r}(x),(3.5)$ is also equivalent to an identity of this type. For similar equivalences in a related context, see $[3$.

\section{Theorem 3.2}

For $r=1,2$, let $F_{r}:(0, \infty) \rightarrow \mathbb{C}$ be continuous functions which are $O\left(x^{-1-\varepsilon}\right)$ as $x \rightarrow 0^{+}$ for every $\varepsilon>0$ and $O\left(x^{-\beta_{r}}\right)$ as $x \rightarrow \infty$, for some $\beta_{r}>1$. Let $\Psi_{r}(s)$ be the Mellin transform of $F_{r}(x)$ defined for $\Re s \in\left(1, \beta_{r}\right)$. Then the following are equivalent:

(a) $\Psi_{1}(s)$ and $\Psi_{2}(s)$ have analytic continuations to $1-\beta_{2}<\Re s<\beta_{1}$ and $1-\beta_{1}<\Re s<$ $\beta_{2}$ respectively except for a finite number of poles in any given strip $\sigma_{1} \leq \Re s \leq \sigma_{2}$, tend to 0 uniformly as $|\Im s| \rightarrow \infty$, and satisfy the functional equation

$$
\Psi_{1}(1-s)=\Psi_{2}(s) \quad \text { for } 1-\beta_{1}<\Re s<\beta_{2} .
$$

(b)

$$
F_{1}(x)=\frac{1}{x} F_{2}\left(\frac{1}{x}\right)+H(x),
$$

where $H(x)$ is a finite series of the form $\sum_{k} a_{k} x^{\mu_{k}}(\log x)^{\nu_{k}}$ with $\mu_{k} \in \mathbb{C}$ and $\nu_{k} \in \mathbb{N}_{0}$.

For the specific form of functional equation (3.5), we take

$$
F_{r}(x)=\sum_{n \in \mathcal{N}_{r}} g_{r}(n x) \quad(r=1,2),
$$

where $\mathcal{N}_{r}$ are two g-integer systems, the $g_{r}(x)$ are as before and satisfy $O\left(x^{-\alpha_{r}}\right)$ and $O\left(x^{\beta_{r}}\right)$ as $x \rightarrow 0^{+}$and $x \rightarrow \infty$ respectively, for some $\alpha_{r}<1<\beta_{r}$. Then $F_{r}(x)$ has the appropriate behaviour at 0 and infinity, and $\Psi_{r}(s)=G_{r}(s) \zeta_{r}(s)$. 
Proof of Theorem 3.2. (a) $\Longrightarrow$ (b). By the inverse Mellin transform for $F_{2}(x)$, we have

$$
F_{2}(x)=\frac{1}{2 \pi i} \int_{(c)} \Psi_{2}(s) x^{-s} d s \quad \text { for any } c \in\left(1, \beta_{2}\right),
$$

where $\int_{(c)}$ denotes $\lim _{T \rightarrow \infty} \int_{c-i T}^{c+i T}$. Hence

$$
\begin{aligned}
\frac{1}{x} F_{2}\left(\frac{1}{x}\right) & =\frac{1}{2 \pi i} \int_{(c)} \Psi_{2}(s) x^{s-1} d s \\
= & \frac{1}{2 \pi i} \int_{(c)} \Psi_{1}(1-s) x^{s-1} d s \\
= & \frac{1}{2 \pi i} \int_{\left(c^{\prime}\right)} \Psi_{1}(s) x^{-s} d s \quad\left(\text { where } c^{\prime}=1-c\right) .
\end{aligned}
$$

On the other hand,

$$
F_{1}(x)=\frac{1}{2 \pi i} \int_{(c)} \Psi_{1}(s) x^{-s} d s \quad \text { for any } c \in\left(1, \beta_{1}\right) .
$$

Moving the contour from the line $\Re s=c$ to $\Re s=c^{\prime}$, we pick up the residues at the poles of $\Psi_{1}(s)$ in the strip $\{s: 0 \leq \Re s \leq 1\}$. By assumption there are only a finite number of these and the residues of the integrand are all of the form $a x^{\mu} P(\log x)$ for some $a, \mu \in \mathbb{C}$ and some polynomial $P(\cdot)$. Moving the contour is permissible here by the assumption that $\Psi_{1}(\sigma+i T) \rightarrow 0$ uniformly as $|T| \rightarrow \infty$. Hence

$$
F_{1}(x)=\frac{1}{x} F_{2}\left(\frac{1}{x}\right)+\sum_{k} a_{k} x^{\mu_{k}}(\log x)^{\nu_{k}}
$$

for some constants $a_{k}, \mu_{k} \in \mathbb{C}$ and $\nu_{k} \in \mathbb{N}_{0}$.

(b) $\Longrightarrow(\mathrm{a})$. For $r=1,2$, we write

$$
\Psi_{r}(s)=\int_{0}^{1} x^{s-1} F_{r}(x) d x+\int_{1}^{\infty} x^{s-1} F_{r}(x) d x=A_{r}(s)+B_{r}(s),
$$

where $A_{r}(s)=\int_{0}^{1} x^{s-1} F_{r}(x) d x$ and $B_{r}(s)=\int_{1}^{\infty} x^{s-1} F_{r}(x) d x$. These integrals converge for $\Re s>1$ and $\Re s<\beta_{r}$ respectively. Now

$$
\begin{aligned}
A_{1}(s) & =\int_{0}^{1} x^{s-1} F_{1}(x) d x=\int_{0}^{1} x^{s-1}\left(\frac{1}{x} F_{2}\left(\frac{1}{x}\right)+H(x)\right) d x \\
& =\int_{1}^{\infty} x^{-s} F_{2}(x) d x+\int_{0}^{1} x^{s-1} H(x) d x \\
& =B_{2}(1-s)+H_{1}(s) .
\end{aligned}
$$

On the other hand,

$$
\begin{aligned}
B_{1}(s) & =\int_{1}^{\infty} x^{s-1} F_{1}(x) d x=\int_{1}^{\infty} x^{s-1}\left(\frac{1}{x} F_{2}\left(\frac{1}{x}\right)+H(x)\right) d x \\
& =\int_{0}^{1} x^{-s} F_{2}(x) d x+\int_{1}^{\infty} x^{s-1} H(x) d x \\
& =A_{2}(1-s)+H_{2}(s) .
\end{aligned}
$$


Where do (3.7) and (3.8) hold? The functions $H_{1}(s)$ and $H_{2}(s)$ are actually rational functions as shown below and hence have meromorphic continuations to $\mathbb{C}$. Now $A_{1}(s)$ is analytic for $\Re s>1$ while $B_{2}(1-s)$ is analytic for $\Re s>1-\beta_{2}$. Thus $A_{1}(s)$ has a meromorphic continuation for $\Re s>1-\beta_{2}$ and (3.7) holds for $\Re s>1-\beta_{2}$. Similarly, (3.8) holds for $\Re s<\beta_{1}$. These provide the required continuations of $\Psi_{1}(s)$ and $\Psi_{2}(s)$. In particular, for $1-\beta_{2}<\Re s<\beta_{1}$, (3.7) and (3.8) hold simultaneously and together give

$$
A_{1}(s)+B_{1}(s)=A_{2}(1-s)+B_{2}(1-s)+H_{1}(s)+H_{2}(s),
$$

i.e.

$$
\Psi_{1}(s)=\Psi_{2}(1-s)+H_{1}(s)+H_{2}(s) .
$$

We proceed to show that $H_{1}(s)$ and $H_{2}(s)$ are rational functions such that $H_{1}(s)+H_{2}(s)=$ 0 . Indeed, we have

$$
\begin{aligned}
H_{1}(s) & =\int_{0}^{1} x^{s-1} H(x) d x=\sum_{k} a_{k} \int_{0}^{1} x^{s+\mu_{k}-1}(\log x)^{\nu_{k}} d x \quad\left(x=e^{-t}\right) \\
& =\sum_{k} a_{k}(-1)^{\nu_{k}} \int_{0}^{\infty} t^{\nu_{k}} e^{-\left(s+\mu_{k}\right) t} d t,
\end{aligned}
$$

the integral converging whenever $\Re\left(s+\mu_{k}\right)>0$ for all $k$. For $H_{2}(s)$ we have

$$
\begin{aligned}
H_{2}(s) & =\int_{1}^{\infty} x^{s-1} H(x) d x=\sum_{k} a_{k} \int_{1}^{\infty} x^{s+\mu_{k}-1}(\log x)^{\nu_{k}} d x \quad\left(x=e^{t}\right) \\
& =\sum_{k} a_{k} \int_{0}^{\infty} t^{\nu_{k}} e^{\left(s+\mu_{k}\right) t} d t,
\end{aligned}
$$

the integral converging whenever $\Re\left(s+\mu_{k}\right)<0$ for all $k$. But for $m$ a non-negative integer and $\alpha \in \mathbb{C}$ with positive real part, $\int_{0}^{\infty} t^{m} e^{-\alpha t} d t=m ! \alpha^{-m-1}$. Thus

$$
\begin{gathered}
H_{1}(s)=\sum_{k} \frac{a_{k}(-1)^{\nu_{k}} \nu_{k} !}{\left(s+\mu_{k}\right)^{\nu_{k}+1}}, \quad \text { and } \\
H_{2}(s)=\sum_{k} \frac{a_{k} \nu_{k} !}{\left(-\left(s+\mu_{k}\right)\right)^{\nu_{k}+1}}=\sum_{k} \frac{a_{k}(-1)^{\nu_{k}+1} \nu_{k} !}{\left(s+\mu_{k}\right)^{\nu_{k}+1}}=-H_{1}(s),
\end{gathered}
$$

as required.

Finally, we show that $\left|\Psi_{r}(s)\right| \rightarrow 0$ uniformly as $|\Im s| \rightarrow \infty$, for $r=1,2$. By the functional equation, it suffices to show this for $\left|\Psi_{1}(s)\right|$. Let $s=\sigma+i t$. We have

$$
\Psi_{1}(s)=A_{1}(s)+B_{1}(s)=B_{2}(1-s)+B_{1}(s)+H_{1}(s) .
$$

Now $\left|H_{1}(s)\right|=O(1 /|t|)$, while

$$
\left|B_{1}(\sigma+i t)\right|=\left|\int_{1}^{\infty} x^{\sigma-1+i t} F_{1}(x) d x\right|=\left|\int_{1}^{\infty} x^{\sigma-1} F_{1}(x) e^{i t \log x} d x\right| .
$$


The integral $\int_{1}^{\infty} x^{\sigma-1} F_{1}(x) d x$ converges uniformly whenever $\sigma \leq \beta_{1}-\delta$ for any $\delta>0$; hence by the Riemann-Lebesgue Theorem, it follows that the RHS integral tends to 0 as $|t| \rightarrow \infty$ uniformly for $\sigma \leq \beta_{1}-\delta$. Similarly, $\left|B_{2}(1-\sigma+i t)\right| \rightarrow 0$ uniformly for $\sigma \geq 1-\beta_{2}+\delta$. Hence $\left|\Psi_{1}(\sigma+i t)\right| \rightarrow 0$ uniformly as $|t| \rightarrow \infty$ for $1-\beta_{2}+\delta \leq \sigma \leq \beta_{1}-\delta$.

Remark. In a work in preparation [30], the second author and R. Nest have obtained (under hypotheses similar to those of Proposition 3.1) a generalised functional equation for $\zeta(s)$. The latter connects a suitable completion of the Beurling zeta function $\zeta(s)$ associated to the g-prime system $\mathcal{P}$ to that of $\zeta^{*}(s)$, associated to a 'dual system' $\mathcal{P}^{*}$ (which involves in general a continuous g-prime system); ${ }^{13}$ this establishes part of a conjecture formulated in [27], §4.4. (see $\$ 1.3$ above). We note that the context of [30] is not restricted to Beurling zeta functions and applies, in particular, to zeta functions associated with quasicrystals.

\section{§4. Partial orders on $\mathcal{N}$}

We have of course a great deal of freedom when choosing a system of generalised primes. Each $p_{j}$ can be chosen arbitrarily as long as it is larger than $p_{j-1}$. Every choice of system $\mathcal{P}$ results in an ordering of $\mathcal{N}$. For two different systems, we would (in general) expect to have two different orderings on the corresponding $\mathcal{N}$ s. For example, in one case we might have $p_{1}^{2}<p_{2}$, while in the next maybe $p_{1}^{2}>p_{2}$. This raises the following questions:

(a) Are orderings on $\mathcal{N}$ uniquely determined by the choice of $\mathcal{P}$ ?

(b) Given that we know the ordering on $\mathcal{N}$, can we reconstruct $\mathcal{P}$ ?

The answer to (a) is negative for an obvious reason: having chosen $\mathcal{P}$, then for any $\lambda>0$, the system $\mathcal{P}^{\lambda}=\left\{p^{\lambda}: p \in \mathcal{P}\right\}$ produces the same ordering in its generalised integers as that of $\mathcal{P}$ - they are just the $\lambda^{\text {th }}$ powers of the integers in $\mathcal{N}$. We show below that this is the only case where this happens; that is, two essentially different prime systems produce different orderings (see also [14 for similar results). In the following, we set $\mathcal{N}^{\lambda}=\left\{n^{\lambda}: n \in \mathcal{N}\right\}$.

\section{Theorem 4.1}

Let $\mathcal{P}_{1}$ and $\mathcal{P}_{2}$ be two generalised prime systems with generalised integers $\mathcal{N}_{1}$ and $\mathcal{N}_{2}$ respectively. If the orderings of $\mathcal{N}_{1}$ and $\mathcal{N}_{2}$ coincide, then $\mathcal{P}_{1}=\mathcal{P}_{2}^{\lambda}$ for some $\lambda>0$ and hence $\mathcal{N}_{1}=\mathcal{N}_{2}^{\lambda}$.

Proof. Denote the primes in $\mathcal{P}_{1}$ by $p_{1}, p_{2}, \ldots$, and those in $\mathcal{P}_{2}$ by $q_{1}, q_{2}, \ldots$ Let $k, m \in \mathbb{N}$. Then $p_{k}^{m} \in\left[p_{1}^{n}, p_{1}^{n+1}\right.$ ) for some $n \in \mathbb{N}$. Since $\mathcal{N}_{2}$ has the same ordering as $\mathcal{N}_{1}$ we also have $q_{k}^{m} \in\left[q_{1}^{n}, q_{1}^{n+1}\right)$. Taking logs gives

$$
n \leq \frac{m \log p_{k}}{\log p_{1}}<n+1 \quad \text { and } \quad n \leq \frac{m \log q_{k}}{\log q_{1}}<n+1
$$

\footnotetext{
${ }^{13}$ Determining when these g-prime systems have an infinite discrete part is an interesting and difficult problem that is far from resolved at this stage.
} 
i.e. $n=\left[\frac{m \log p_{k}}{\log p_{1}}\right]=\left[\frac{m \log p_{k}}{\log p_{1}}\right]$. It follows that

$$
\frac{m \log p_{k}}{\log p_{1}}-1<n \leq \frac{m \log q_{k}}{\log q_{1}}<n+1 \leq \frac{m \log p_{k}}{\log p_{1}}+1
$$

and hence

$$
\frac{\log p_{k}}{\log p_{1}}-\frac{1}{m}<\frac{\log q_{k}}{\log q_{1}}<\frac{\log p_{k}}{\log p_{1}}+\frac{1}{m} .
$$

This holds for all $m$. Letting $m \rightarrow \infty$ gives

$$
\frac{\log p_{k}}{\log p_{1}}=\frac{\log q_{k}}{\log q_{1}}
$$

i.e. $p_{k}=q_{k}^{\lambda}$ where $\lambda=\frac{\log p_{1}}{\log q_{1}}$, and hence $\mathcal{P}_{1}=\mathcal{P}_{2}^{\lambda}$.

The above theorem actually shows that the order is determined uniquely (up to a constant) by the way the powers of the generalised primes are ordered. It is therefore of importance to consider the set of powers of generalised primes. We shall denote it by $\mathcal{Q}$; i.e.

$$
\mathcal{Q}=\left\{p^{n}: p \in \mathcal{P}, n \in \mathbb{N}\right\}
$$

The set $\mathcal{Q}$ is isomorphic to $\mathbb{N}^{2}=\mathbb{N} \times \mathbb{N}$ via the isomorphism: $p_{m}^{n} \mapsto(m, n)$. Furthermore, the ordering on $\mathcal{Q}$ induces an order on $\mathbb{N}^{2}$. Such an order necessarily satisfies the following three axioms:

A1 $(m, n) \leq\left(m^{\prime}, n^{\prime}\right)$ whenever both $m \leq m^{\prime}$ and $n \leq n^{\prime}$, with strict inequality if $n<n^{\prime}$.

A2 $(m, n) \leq\left(m^{\prime}, n^{\prime}\right)$ implies $(m, k n) \leq\left(m^{\prime}, k n^{\prime}\right)$ for every $k \in \mathbb{N}$, with strict inequality if $(m, n)<\left(m^{\prime}, n^{\prime}\right)$.

A3 Finiteness: (i) for all $n \in \mathbb{N}$, there exists $k \in \mathbb{N}$ such that $(1, n)<(k, 1)$ and

(ii) for all $m \in \mathbb{N}$, there exists $l \in \mathbb{N}$ such that $(m, 1)<(1, l)$.

On the other hand, we show below in Theorem 4.3 that for any order on $\mathbb{N}^{2}$ satisfying axioms A1, A2, and A3, there is a generalised prime system $\mathcal{P}$ for which $\mathcal{Q}$ has the same ordering.

First we need the following lemma:

\section{Lemma 4.2}

Let $f: \mathbb{N} \rightarrow \mathbb{R}$ be such that for all $m, n \in \mathbb{N}$

$$
\left|\frac{f(m n)}{m n}-\frac{f(n)}{n}\right| \leq \frac{1}{n} .
$$

Then $\lim _{n \rightarrow \infty} \frac{f(n)}{n}$ exists. 
Proof. By putting $n=1$ in (4.1), it follows that $\frac{f(n)}{n}$ is bounded. Let

$$
\alpha=\liminf _{n \rightarrow \infty} \frac{f(n)}{n} .
$$

By definition of $\alpha$, there exists a sequence $n_{k}$ tending to infinity with $k$ such that

$$
\frac{f\left(n_{k}\right)}{n_{k}} \rightarrow \alpha \quad \text { as } k \rightarrow \infty
$$

Hence, for fixed $m \in \mathbb{N}$, we have $\frac{f\left(m n_{k}\right)}{m n_{k}} \rightarrow \alpha$ as $k \rightarrow \infty$, since

$$
|\frac{f\left(m n_{k}\right)}{m n_{k}}-\underbrace{\frac{f\left(n_{k}\right)}{n_{k}}}_{\rightarrow \alpha}| \leq \underbrace{\frac{1}{n_{k}}}_{\rightarrow 0} .
$$

Now fix $n \in \mathbb{N}$, put $m=n_{k}$ in (4.1) and let $k \rightarrow \infty$. Then

$$
|\underbrace{\frac{f\left(n n_{k}\right)}{n n_{k}}}_{\rightarrow \alpha}-\frac{f(n)}{n}| \leq \frac{1}{n} \text {. }
$$

Thus

$$
\left|\frac{f(n)}{n}-\alpha\right| \leq \frac{1}{n}
$$

and the result follows.

\section{Theorem 4.3}

Given an order on $\mathbb{N}^{2}$ satisfying axioms A1-A3, there exists a generalised prime system $\mathcal{P}$ which induces this order.

Proof. Fix $k \in \mathbb{N}$. For $n \in \mathbb{N}$, let $f_{k}(n)$ be the unique positive integer for which

$$
\left(1, f_{k}(n)\right) \leq(k, n)<\left(1, f_{k}(n)+1\right) .
$$

This exists on account of A2 and A3, and is unique by A1. Hence, replacing $n$ by $m n$ $(m \in \mathbb{N})$, we have

$$
\left(1, f_{k}(m n)\right) \leq(k, m n)<\left(1, f_{k}(m n)+1\right) .
$$

On the other hand, A2 implies that

$$
\left(1, m f_{k}(n)\right) \leq(k, m n)<\left(1, m\left(f_{k}(n)+1\right)\right) .
$$

(4.2) and (4.3) give

$$
\begin{aligned}
& \left(1, f_{k}(m n)\right)<\left(1, m\left(f_{k}(n)+1\right)\right), \text { and } \\
& \left(1, m f_{k}(n)\right)<\left(1, f_{k}(m n)+1\right) .
\end{aligned}
$$


Thus $f_{k}(m n)<m f_{k}(n)+m$ and $m f_{k}(n)<f_{k}(m n)+1$. Combining these and dividing through by $m n$ gives

$$
\frac{f_{k}(n)}{n}-\frac{1}{m n}<\frac{f_{k}(m n)}{m n}<\frac{f_{k}(n)}{n}+\frac{1}{n},
$$

and so $f_{k}(n)$ satisfies condition (4.1) of Lemma 4.2. Hence $\frac{f_{k}(n)}{n} \rightarrow \alpha_{k}$ for some $\alpha_{k}$. Also, $\alpha_{k}$ increases with $k$, since $f_{k}(n)$ does.

Choose $p_{1}>1$ arbitrarily, and define g-primes by $p_{k}=p_{1}^{\alpha_{k}}$ (note that $\alpha_{1}=1$ ). This gives a system of generalised primes which induces the same order. Indeed, let $m \rightarrow \infty$ in (4.4). Then $f_{k}(n) \leq n \alpha_{k} \leq f_{k}(n)+1$. Now if $p_{m}^{n}<p_{m^{\prime}}^{n^{\prime}}\left(\right.$ i.e. $\left.n \alpha_{m}<n^{\prime} \alpha_{m^{\prime}}\right)$, then

$$
f_{m}(n) \leq n \alpha_{m}<n^{\prime} \alpha_{m^{\prime}} \leq f_{m^{\prime}}\left(n^{\prime}\right)+1 .
$$

Since, $f_{m}(n)$ and $f_{m^{\prime}}\left(n^{\prime}\right)$ are integers, this implies $f_{m}(n) \leq f_{m^{\prime}}\left(n^{\prime}\right)$, i.e. $(m, n) \leq\left(m^{\prime}, n^{\prime}\right)$. But if $(m, n)=\left(m^{\prime}, n^{\prime}\right)$, then $(m, k n)=\left(m^{\prime}, k n^{\prime}\right)$ for all $k \in \mathbb{N}$ by A2. Hence $\frac{f_{m}(k n)}{k}=$ $\frac{f_{m^{\prime}}\left(k n^{\prime}\right)}{k}$ and, letting $k \rightarrow \infty$, we have $n \alpha_{m}=n^{\prime} \alpha_{m^{\prime}}$, i.e. $p_{m}^{n}=p_{m^{\prime}}^{n^{\prime}}$.

This shows that $p_{m}^{n}<p_{m^{\prime}}^{n^{\prime}}$ implies $(m, n)<\left(m^{\prime}, n^{\prime}\right)$.

Remark. In the light of the results obtained in the present section, it would be interesting to develop a suitable theory of generalised valuations for g-integer systems. We leave this problem for future investigations.

\section{References}

[1] T. M. Apostol, Mathematical Analysis, Addison-Wesley, Massachusetts, 1973.

[2] P. T. Bateman and H. G. Diamond, Asymptotic distribution of Beurling's generalised prime numbers, in: Studies in Number Theory 6, Prentice-Hall, 1969, pp. 152-212.

[3] B. C. Berndt, Identities involving the coefficients of a class of Dirichlet series I, Trans. Amer. Math. Soc. 137 (1969), 345-359.

[4] A. Beurling, Analyse de la loi asymptotique de la distribution des nombres premiers généralisés, I, Acta Math. 68 (1937), 255-291.

[5] S. Bochner, Some properties of modular relations, Ann. of Math. 53 No. 2 (1951), 332-363.

[6] A. Connes, Noncommutative Geometry, Academic Press, New York, 1994.

[7] J. B. Conrey and A. Ghosh, On the Selberg class of Dirichlet series: small degrees, Duke Math. J. 72 (1993), 673-693.

[8] H. G. Diamond, The prime number theorem for Beurling generalised numbers, J. Number Theory 1 (1969), 200-207.

[9] H. G. Diamond, Asymptotic distribution of Beurling generalised integers, Illinois J. Math. 14 (1970), 12-28. 
[10] H. G. Diamond, A set of generalised numbers showing Beurling's theorem to be sharp, Illinois J. Math. 14 (1970), 29-34.

[11] H. G. Diamond, When do Beurling generalised integers have a density?, J. Reine Angew. Math. 295 (1977), 22-39.

[12] H. Diamond, H. Montgomery and U. Vorhauer, Beurling primes with large oscillation. Preprint, 2003.

[13] H. M. Edwards, Riemann's Zeta Function, Academic Press, New York, 1974. (Reprinted by Dover Publ., 2001.)

[14] L. Fuchs, Partially Ordered Algebraic Systems, International Series of Monographs in Pure and Applied Mathematics 28, Pergamon Press, 1963.

[15] R. S. Hall, The prime number theorem for generalised primes, J. Number Theory 4 (1972), 313-320.

[16] R. S. Hall, Theorems about Beurling's generalised primes and the associated zeta function, Ph.D. Thesis, University of Illinois, Urbana, 1967.

[17] C. Q. He and M. L. Lapidus, Generalized Minkowski content, spectrum of fractal drums, fractal strings and the Riemann zeta function, Memoirs Amer. Math. Soc., No. 608, 127 (1997), 1-97.

[18] T. W. Hilberdink, Some connections between Bernoulli convolutions and analytic number theory, in: Fractal Geometry and Number Theory: A Jubilee of Benoit Mandelbrot (M. L. Lapidus and M. van Frankenhuysen, eds.), Proc. Sympos. Pure Math., Amer. Math. Soc., Providence R. I., 2004 (to appear).

[19] M. N. Huxley, Exponential sums and lattice points, Proc. London Math. Soc. 60 (1990), 471-475.

[20] A. E. Ingham, The Distribution of Prime Numbers, Cambridge Tracts in Math. 30, Second edition (reprinted from the 1932 edition), Cambridge University Press, 1990.

[21] J. Knopfmacher, Abstract Analytic Number Theory, Dover, 1990.

[22] J. C. Lagarias, Beurling Generalised Integers with the Delone Property, Forum Math. 11 (1999), 295-312.

[23] E. Landau, Neuer Beweis des Primzahlsatzes und Beweis des Primidealsatzes, Math. Ann. 56 (1903), 645-670.

[24] M. L. Lapidus, Fractal drums, inverse spectral problems for elliptic operators and a partial resolution of the Weyl-Berry conjecture, Trans. Amer. Math. Soc. 325 (1991), 465-529.

[25] M. L. Lapidus, Vibrations of fractal drums, the Riemann hypothesis, waves in fractal media, and the Weyl-Berry conjecture, in: Ordinary and Partial Differential Equations (B. D. Sleeman and R. J. Jarvis, eds.), vol. IV, Proc. Twelfth Internat. Conf. (Dundee, Scotland, UK, June 1992), Pitman Research Notes in Math. Series, vol. 289, Longman Scientific and Technical, London, 1993, pp. 126-209. 
[26] M. L. Lapidus, T-duality, functional equation, and noncommutative stringy spacetime, in: New Interactions of Mathematics with the Natural Sciences and the Humanities (L. Boi, ed.), Springer-Verlag, Berlin, 2003, pp. 1-92 (in press).

[27] M. L. Lapidus, In Search of the Riemann Zeros: Strings, fractal membranes, and noncommutative spacetimes, Book in preparation, 2003, 365+(xii) pp.

[28] M. L. Lapidus and H. Maier, The Riemann hypothesis and inverse spectral problems for fractal strings, J. London Math. Soc. (2) 52 (1995), 15-34.

[29] M. L. Lapidus and R. Nest, Fractal membranes and the second quantization of fractal strings, in preparation.

[30] M. L. Lapidus and R. Nest, Functional equations for zeta functions associated with quasicrystals and fractal membranes, in preparation.

[31] M. L. Lapidus and C. Pomerance, The Riemann zeta-function and the one-dimensional Weyl-Berry conjecture for fractal drums, Proc. London Math. Soc. (3) 66 (1993), 41-69.

[32] M. L. Lapidus and C. Pomerance, Counterexamples to the modified Weyl-Berry conjecture on fractal drums, Proc. Cambridge Philos. Soc. 199 (1996), 167-178.

[33] M. L. Lapidus and M. van Frankenhuysen, Fractal Geometry and Number Theory: Complex dimensions of fractal strings and zeros of zeta functions, Birkhäuser, Boston, 2000. (Second revised and enlarged edition to appear in 2004.)

[34] M. L. Lapidus and M. van Frankenhuysen, Fractality, self-similarity and complex dimensions, in: Fractal Geometry and Number Theory: A Jubilee of Benoit Mandelbrot (M. L. Lapidus and M. van Frankenhuysen, eds.), Proc. Sympos. Pure Math., Amer. Math. Soc., Providence, R. I., 2004 (to appear).

[35] P. Malliavin, Sur le reste de la loi asymptotique de répartition des nombres premiers généralisés de Beurling, Acta Math. 106 (1961), 281-298.

[36] B. Nyman, A general prime number theorem, Acta Math. 81 (1949), 299-307.

[37] M. Ram Murty, Selberg's conjectures and Artin L-functions, Bull. Amer. Math. Soc. 31 (1994), 1-14.

[38] C. Ryavec, The analytic continuation of Euler products with applications to asymptotic formulae, Illinois J. Math. 17 (1973), 608-616.

[39] A. Selberg, Old and new conjectures about a class of Dirichlet series, in: A. Selberg: Collected Papers, vol. II, Springer-Verlag, New York, 1991, pp. 47-63.

[40] E. C. Titchmarsh, The Theory of Functions, Second edition, Oxford University Press, 1986.

[41] E. C. Titchmarsh, The Theory of the Riemann Zeta-function, Second edition, Oxford University Press, 1986.

[42] Wen-Bin Zhang, Density and O-Density of Beurling generalised integers, J. Number Theory 30 (1988), 120-139. 
[43] Wen-Bin Zhang, A generalization of Halász's theorem to Beurling's generalised integers and its application, Illinois J. Math. 31 (1987), 645-664.

Titus W. Hilberdink, Department of Mathematics, University of Reading, Whiteknights, PO Box 220, Reading RG6 6AX, UK.

E-mail address: t.w.hilberdink@reading.ac.uk

Michel L. Lapidus, Department of Mathematics, University of California, Riverside, CA 92521-0135, USA.

E-mail: lapidus@math.ucr.edu 